\title{
SOME CONSTRUCTIONS OF MODULAR FORMS FOR THE WEIL REPRESENTATION OF $\mathrm{SL}_{2}(\mathbb{Z})$
}

\author{
NILS R. SCHEITHAUER
}

\begin{abstract}
Modular forms for the Weil representation of $\mathrm{SL}_{2}(\mathbb{Z})$ play an important role in the theory of automorphic forms on orthogonal groups. In this paper we give some explicit constructions of these functions. As an application, we construct new examples of generalized Kac-Moody algebras whose denominator identities are holomorphic automorphic products of singular weight. They correspond naturally to the Niemeier lattices with root systems $D_{12}^{2}, E_{8}^{3}$ and to the Leech lattice.
\end{abstract}

\section{$\S 1$. Introduction}

The singular theta correspondence (see [3]; see also [5]) is a map from modular forms for the Weil representation of $\mathrm{SL}_{2}(\mathbb{Z})$ to automorphic forms on orthogonal groups. More precisely, let $L$ be an even lattice of signature $(n, 2), n>2$ even with discriminant form $D$, and let $F$ be a modular form for the Weil representation of $\mathrm{SL}_{2}(\mathbb{Z})$ on $\mathbb{C}[D]$ of weight $(2-n) / 2$ which is holomorphic on the upper half-plane and has integral principal part. Then the integral

$$
\int_{\mathcal{F}} F(\tau) \bar{\theta}(Z, \tau) y \frac{d x d y}{y^{2}}
$$

where $\mathcal{F}$ is the standard fundamental domain of $\mathrm{SL}_{2}(\mathbb{Z})$ on the upper halfplane and $\theta$ is the Siegel theta function of $L$, can be regularized. Let $\Psi(Z)$ be the exponential of the regularized value. Then $\Psi(Z)$ is an automorphic form for a subgroup of $O(L)$ which has nice product expansions at the rational 0 -dimensional cusps. The function $\Psi(Z)$ is called the automorphic product associated to $F$. The weight of $\Psi$ is determined by the constant coefficient of $F_{0}$, and its divisor is determined by the principal part of $F$.

The smallest possible weight of a nonconstant holomorphic automorphic form on $O_{n, 2}(\mathbb{R})$ is given by $(n-2) / 2$. Holomorphic automorphic forms

Received December 9, 2013. Revised June 20, 2014. Accepted August 13, 2014.

2010 Mathematics Subject Classification. Primary 11F27; Secondary 11F22, 17 B65. 
of this so-called singular weight are particularly interesting because their Fourier coefficients are supported only on isotropic vectors. It has turned out that the denominator identities of infinite-dimensional Lie algebras are sometimes holomorphic automorphic products of singular weight. These Lie algebras describe strings moving on suitable orbifolds and they seem to be very rare (see [12]). So far there are only twelve known examples (see [13]).

In this article, we give some explicit constructions of modular forms for the Weil representation of $\mathrm{SL}_{2}(\mathbb{Z})$. As an application, we construct three new generalized Kac-Moody algebras whose denominator identities are holomorphic automorphic products of singular weight. In contrast to the previous examples, the corresponding vector-valued modular forms are not symmetric under $O(D)$, which makes their construction more difficult.

We describe the results in more detail.

First, we show how scalar-valued modular forms on congruence subgroups induce modular forms for the Weil representation of $\mathrm{SL}_{2}(\mathbb{Z})$. For $\Gamma_{1}(N)$, the result is as follows (see Theorems 3.1 and 3.7).

Let $D$ be a discriminant form of even signature and level dividing $N$, and let $\rho_{D}$ be the Weil representation of $\mathrm{SL}_{2}(\mathbb{Z})$ on $\mathbb{C}[D]$. Let $\gamma \in D$, and let $f$ be a modular form on $\Gamma_{1}(N)$ with character $\chi_{\gamma}$. Then

$$
F_{\Gamma_{1}(N), f, \gamma}=\left.\sum_{M \in \Gamma_{1}(N) \backslash \Gamma} f\right|_{M} \rho_{D}\left(M^{-1}\right) e^{\gamma}
$$

is a modular form for $\rho_{D}$. Every modular form for $\rho_{D}$ is a linear combination of such functions. The function $F_{\Gamma_{1}(N), f, \gamma}$ can be written as a sum $\sum F_{s}$ over the cusps of $\Gamma_{1}(N)$, where

$$
\begin{aligned}
F_{s}= & \xi\left(M^{-1}\right) \frac{\sqrt{\left|D_{c}\right|}}{\sqrt{|D|}} \sum_{\mu \in a \gamma+D^{c *}} e\left(d(\mu-a \gamma)_{c}^{2} / 2\right) e(b \mu \gamma) e\left(-a b \gamma^{2} / 2\right) \\
& \times t g_{m t, j_{\mu}}\left\{e^{\mu}+(-1)^{k} e(\operatorname{sign}(D) / 4) e^{-\mu}\right\}
\end{aligned}
$$

if $N>2$ and $s$ is regular, and similarly in the other cases.

There are analogous results for the congruence subgroups $\Gamma(N)$ and $\Gamma_{0}(N)$. However, the liftings are not equivalent. In Section 6 we give an example of a modular form for the Weil representation which is not induced from $\Gamma_{0}(N)$.

Next, we describe an induction from the isotropic subgroups of a discriminant form (see Theorem 4.1). 
Let $D$ be a discriminant form of even signature, and let $H$ be an isotropic subgroup of D. Let $F_{D_{H}}=\sum_{\gamma \in D_{H}} F_{D_{H}, \gamma} e^{\gamma}$ be a modular form for the Weil representation of the discriminant form $D_{H}=H^{\perp} / H$. Then

$$
F=\sum_{\gamma \in H^{\perp}} F_{D_{H}, \gamma+H} e^{\gamma}
$$

is a modular form for $\rho_{D}$.

Modular forms which are induced in this way can be considered as oldforms.

Let $D$ be a discriminant form of even signature and level dividing $N$. We have seen that all modular forms for $\rho_{D}$ are induced from $\Gamma(N)$ or $\Gamma_{1}(N)$. However, calculating the corresponding liftings is often laborious because the indices of these groups in $\mathrm{SL}_{2}(\mathbb{Z})$ are rather large. We give a natural sufficient condition for a modular form for $\rho_{D}$ to be induced from $\Gamma_{0}(N)$ (see Proposition 5.3 and Theorem 5.4).

Let $D$ be a discriminant form of square-free level $N$, and let $F=\sum F_{\gamma} e^{\gamma}$ be a modular form for $\rho_{D}$ which is invariant under $O(D)$. Then the complex vector space $W$ spanned by the components $F_{\gamma}, \gamma \in D$ is generated by the functions $\left.F_{0}\right|_{M}, M \in \mathrm{SL}_{2}(\mathbb{Z})$. Let $W_{0}$ be the subspace of $W$ with $T$ eigenvalue $e(0)$. Then the map

$$
\begin{aligned}
\Phi: W_{0} & \longrightarrow W_{0}, \\
f & \longmapsto 0 \text {-component of } F_{\Gamma_{0}(N), f, 0}
\end{aligned}
$$

is a bijection. In particular, $F=F_{\Gamma_{0}(N), f, 0}$ for a suitable function $f$ in $W_{0}$.

Finally, we use the above results to construct some modular forms for the Weil representation with nonnegative integral coefficients and reflective poles. The theta lifts of these functions have singular weight and give the denominator identities of some new generalized Kac-Moody algebras. The simplest example is the following (see Section 6).

Let $N$ be the Niemeier lattice with root system $E_{8}^{3}$. Let $g$ be a permutation of the three $E_{8}$-components of order 3 . Then the fixed-point sublattice $N^{g}$ of $g$ is isomorphic to $\sqrt{3} E_{8}$, and the orthogonal complement $N^{g \perp}$ is isomorphic to $A_{2} \otimes E_{8}$. The theta function $\theta_{N^{g} \perp}$ defines a modular form for the discriminant form of $N^{g}$. This function is invariant under $O\left(N^{g}\right)$ because the centralizer of $g$ in $O(N)$ induces the full orthogonal group of $N^{g}$. Let $L=N^{g} \oplus\left(\begin{array}{rr}-2 & 3 \\ 3 & 0\end{array}\right)$. Then $\theta_{N^{g} \perp}$ induces a modular form on L. Denote the quotient of this form by the invariant $3 \Delta$ by $F_{\theta_{N} \perp} / 3 \Delta$. Define $\eta_{g}(\tau)=\eta(3 \tau)^{8}$. 
Then the sum

$$
F=F_{\theta_{N} \perp / 3 \Delta}+\frac{1}{3} F_{\Gamma_{0}(9), 1 / \eta_{g}, 0}
$$

is a modular form for the Weil representation of $L \oplus I I_{1,1}$ with nonnegative integral coefficients and reflective poles. The theta lift of $F$ is holomorphic, has singular weight, and is up to a constant given by

$$
\begin{aligned}
& e((\rho, Z)) \prod_{\alpha \in L^{\prime+}}(1-e((\alpha, Z)))^{\left[F_{\alpha+L}\right]\left(-\alpha^{2} / 2\right)} \\
& \quad=\sum_{w \in W} \operatorname{det}(w) e((w \rho, Z)) \prod_{n>0}(1-e((3 n w \rho, Z)))^{8} .
\end{aligned}
$$

This is the denominator identity of a generalized Kac-Moody algebra with root lattice $L^{\prime}$ whose multiplicities and simple roots can be described easily.

There are similar examples for the Niemeier lattice with root system $D_{12}^{2}$ and for the Leech lattice.

Our article is organized as follows.

In Section 2 we recall some properties of modular forms for the Weil representation of $\mathrm{SL}_{2}(\mathbb{Z})$.

Section 3 describes the liftings from modular forms on congruence subgroups to modular forms for the Weil representation of $\mathrm{SL}_{2}(\mathbb{Z})$.

In Section 4, we show that a modular form for the Weil representation of an even lattice induces a modular form for the Weil representation of any finite index sublattice.

In Section 5, we show that symmetric modular forms on discriminant forms of square-free level $N$ are induced from $\Gamma_{0}(N)$.

Finally, we use the above methods to construct some new generalized Kac-Moody algebras with automorphic denominator identity. These Lie algebras correspond naturally to the Niemeier lattices with root systems $D_{12}^{2}$ and $E_{8}^{3}$ and to the Leech lattice.

\section{§2. Modular forms for the Weil representation of $\mathrm{SL}_{2}(\mathbb{Z})$}

In this section we recall some properties of modular forms for the Weil representation of $\mathrm{SL}_{2}(\mathbb{Z})$ from [13].

Let $D$ be a discriminant form with quadratic form $D \rightarrow \mathbb{Q} / \mathbb{Z}, \gamma \mapsto \gamma^{2} / 2$ (see also [9], [6]). The level of $D$ is the smallest positive integer $N$ such that $N \gamma^{2} / 2=0 \bmod 1$ for all $\gamma \in D$. 
Let $c$ be an integer. Then $c$ acts by multiplication on $D$ and we have an exact sequence $0 \rightarrow D_{c} \rightarrow D \rightarrow D^{c} \rightarrow 0$, where $D_{c}$ is the kernel and $D^{c}$ the image of this map. The group $D^{c}$ is the orthogonal complement of $D_{c}$.

Let $D^{c *}$ be the set of elements $\alpha \in D$ satisfying $c \gamma^{2} / 2+\alpha \gamma=0 \bmod 1$ for all $\gamma \in D_{c}$. Then $D^{c *}$ is a coset of $D^{c}$. When we have chosen a Jordan decomposition of $D$, then there is a canonical coset representative $x_{c}$ of $D^{c *}$ satisfying $2 x_{c}=0$. Write $\alpha \in D^{c *}$ as $\alpha=x_{c}+c \gamma$. Then $\alpha_{c}^{2} / 2=c \gamma^{2} / 2+$ $x_{c} \gamma \bmod 1$ is independent of the choice of $\gamma$. This gives a well-defined map $D^{c *} \rightarrow \mathbb{Q} / \mathbb{Z}, \alpha \mapsto \alpha_{c}^{2} / 2$.

More generally, let $H$ be an isotropic subgroup of $D$. We define $D_{H}^{c *}$ as the set of elements $\alpha \in D$ satisfying $c \gamma^{2} / 2+\alpha \gamma=0 \bmod 1$ for all $\gamma \in$ $c^{-1}(H) \cap H^{\perp}$. Then $D_{H}^{c *}=D^{c *}$ if $H=0$.

Suppose that $D$ has even signature. We define a scalar product on the group ring $\mathbb{C}[D]$ which is linear in the first (and antilinear in the second) variable by $\left(e^{\gamma}, e^{\beta}\right)=\delta^{\gamma \beta}$. Then there is a unitary action of the group $\Gamma=$ $\mathrm{SL}_{2}(\mathbb{Z})$ on $\mathbb{C}[D]$ defined by

$$
\begin{aligned}
& \rho_{D}(T) e^{\gamma}=e\left(-\gamma^{2} / 2\right) e^{\gamma}, \\
& \rho_{D}(S) e^{\gamma}=\frac{e(\operatorname{sign}(D) / 8)}{\sqrt{|D|}} \sum_{\beta \in D} e(\gamma \beta) e^{\beta},
\end{aligned}
$$

where $S=\left(\begin{array}{cc}0 & -1 \\ 1 & 0\end{array}\right)$ and $T=\left(\begin{array}{ll}1 & 1 \\ 0 & 1\end{array}\right)$ are the standard generators of $\Gamma$. This representation is called the Weil representation of $\Gamma$ on $\mathbb{C}[D]$. It commutes with $O(D)$.

Let $M=\left(\begin{array}{ll}a & b \\ c & d\end{array}\right) \in \Gamma$. Then

$$
\rho_{D}(M) e^{\gamma}=\xi \frac{\sqrt{\left|D_{c}\right|}}{\sqrt{|D|}} \sum_{\beta \in D^{c *}} e\left(-a \beta_{c}^{2} / 2\right) e(-b \beta \gamma) e\left(-b d \gamma^{2} / 2\right) e^{d \gamma+\beta}
$$

where $\xi=e(\operatorname{sign}(D) / 4) \prod \xi_{p}$. The local factors $\xi_{p}$ can be expressed by means of the Jordan components of $D$ (see [13]).

Let

$$
F(\tau)=\sum_{\gamma \in D} F_{\gamma}(\tau) e^{\gamma}
$$

be a holomorphic function on the upper half-plane with values in $\mathbb{C}[D]$, and let $k$ be an integer. Then $F$ is a modular form for $\rho_{D}$ of weight $k$ if

$$
F(M \tau)=(c \tau+d)^{k} \rho_{D}(M) F(\tau)
$$


for all $M=\left(\begin{array}{ll}a & b \\ c & d\end{array}\right)$ in $\Gamma$ and $F$ is meromorphic at $\infty$. Note that the transformation formula can be written equivalently as

$$
F=\left.\sum_{\gamma \in D} F_{\gamma}\right|_{M^{-1}} \rho_{D}(M) e^{\gamma}
$$

for all $M \in \Gamma$.

The components of a modular form $F=\sum_{\gamma \in D} F_{\gamma} e^{\gamma}$ for $\rho_{D}$ transform as

$$
\left.F_{\gamma}\right|_{M}=\bar{\xi}\left(M^{-1}\right) \frac{\sqrt{\left|D_{c}\right|}}{\sqrt{|D|}} \sum_{\beta \in D^{c *}} e\left(-d \beta_{c}^{2} / 2\right) e(-b \beta \gamma) e\left(-a b \gamma^{2} / 2\right) F_{a \gamma+\beta},
$$

where $M=\left(\begin{array}{ll}a & b \\ c & d\end{array}\right) \in \Gamma$, and $\xi\left(M^{-1}\right)$ is the above root of unity corresponding to $M^{-1}$.

Classical examples of modular forms transforming under the Weil representation are theta functions. Let $L$ be a positive definite even lattice of even rank $2 k$ with discriminant form $D$. For $\gamma \in D$, define

$$
\theta_{\gamma}(\tau)=\sum_{\alpha \in \gamma+L} q^{\alpha^{2} / 2}
$$

with $q^{\alpha^{2} / 2}=e\left(\tau \alpha^{2} / 2\right)$. Then

$$
\theta=\sum_{\gamma \in D} \theta_{\gamma} e^{\gamma}
$$

is a modular form for the dual Weil representation $\bar{\rho}_{D}$ of weight $k$ which is holomorphic at $\infty$.

Let $D$ and $D^{\prime}$ be two discriminant forms of even signature, and let $i: D \rightarrow$ $D^{\prime}$ be an isomorphism of groups satisfying $i(\gamma)^{2} / 2=-\gamma^{2} / 2$ for all $\gamma \in D$. If $F_{D^{\prime}}=\sum_{\gamma \in D^{\prime}} F_{\gamma} e^{\gamma}$ is a modular form for the dual Weil representation $\bar{\rho}_{D^{\prime}}$, then $F_{D}=\sum_{\gamma \in D} F_{i(\gamma)} e^{\gamma}$ transforms under $\rho_{D}$. For example, if $L$ is an even unimodular lattice and $K$ is a primitive sublattice of $L$ with orthogonal complement $K^{\perp}$, then we have a natural isomorphism $i: D_{K} \rightarrow D_{K^{\perp}}$ such that $i(\gamma)^{2} / 2=-\gamma^{2} / 2$.

\section{$\S 3 . \quad$ Liftings from congruence subgroups}

In this section we describe how modular forms on congruence subgroups induce modular forms on $\Gamma$ transforming under the Weil representation, and we calculate these liftings explicitly. For the congruence subgroup $\Gamma_{0}(N)$, these results are already known (see [13]). We include them here because we need the corresponding formulas later. 
Let $D$ be a discriminant form of even signature, and let $N$ be a positive integer such that the level of $D$ divides $N$. Let $F=\sum_{\gamma \in D} F_{\gamma} e^{\gamma}$ be a modular form for $\rho_{D}$, and let $M=\left(\begin{array}{ll}a & b \\ c & d\end{array}\right) \in \Gamma_{0}(N)$. Then the formula for $\rho_{D}$ gives

$$
\rho_{D}(M) e^{\gamma}=\left(\frac{a}{|D|}\right) e((a-1) \operatorname{oddity}(D) / 8) e\left(-b d \gamma^{2} / 2\right) e^{d \gamma}
$$

and

$$
\left.F_{\gamma}\right|_{M}=\left(\frac{d}{|D|}\right) e((d-1) \operatorname{oddity}(D) / 8) e\left(-a b \gamma^{2} / 2\right) F_{a \gamma}
$$

We define the quadratic Dirichlet character $\chi_{D}: \Gamma_{0}(N) \rightarrow \mathbb{C}^{*}$ by

$$
\chi_{D}\left(\left(\begin{array}{ll}
a & b \\
c & d
\end{array}\right)\right)=\left(\frac{a}{|D|}\right) e((a-1) \operatorname{oddity}(D) / 8)
$$

and for $\gamma \in D$ the character $\chi_{\gamma}: \Gamma_{1}(N) \rightarrow \mathbb{C}^{*}$ with

$$
\chi_{\gamma}\left(\left(\begin{array}{ll}
a & b \\
c & d
\end{array}\right)\right)=e\left(-b \gamma^{2} / 2\right)
$$

Then $F_{0}$ is a modular form on $\Gamma_{0}(N)$ of character $\chi_{D}$, and $F_{\gamma}$ is a modular form on $\Gamma_{1}(N)$ with character $\chi_{\gamma}$. Conversely, we have the following result.

THEOREM 3.1. Let $D$ be a discriminant form of even signature and level dividing $N$.

(i) Let $f$ be a scalar-valued modular form on $\Gamma_{0}(N)$ of weight $k$ and character $\chi_{D}$, and let $H$ be an isotropic subset of $D$ which is invariant under $(\mathbb{Z} / N \mathbb{Z})^{*}$ as a set. Then

$$
F_{\Gamma_{0}(N), f, H}=\left.\sum_{M \in \Gamma_{0}(N) \backslash \Gamma} \sum_{\gamma \in H} f\right|_{M} \rho_{D}\left(M^{-1}\right) e^{\gamma}
$$

is a modular form for $\rho_{D}$ of weight $k$ which is invariant under the automorphisms of the discriminant form that stabilize $H$ as a set.

(ii) Let $\gamma \in D$, and let $f$ be a scalar-valued modular form for $\Gamma_{1}(N)$ of weight $k$ and character $\chi_{\gamma}$. Then

$$
F_{\Gamma_{1}(N), f, \gamma}=\left.\sum_{M \in \Gamma_{1}(N) \backslash \Gamma} f\right|_{M} \rho_{D}\left(M^{-1}\right) e^{\gamma}
$$

is a modular form for $\rho_{D}$ of weight $k$ which is invariant under the stabilizer of $\gamma$ in $O(D)$.

(iii) Finally, if $f$ is a scalar-valued modular form on $\Gamma(N)$ of weight $k$ and $\gamma \in D$, then 


$$
F_{\Gamma(N), f, \gamma}=\left.\sum_{M \in \Gamma(N) \backslash \Gamma} f\right|_{M} \rho_{D}\left(M^{-1}\right) e^{\gamma}
$$

is a modular form for $\rho_{D}$ of weight $k$ which is invariant under the stabilizer of $\gamma$ in $O(D)$.

Proof. We have to show that the liftings are well defined, transform correctly under $\Gamma$, and have the stated symmetries. We describe this in the second case. The other cases are analogous.

For $M \in \Gamma$, we define the function $F_{M}=\left.f\right|_{M} \rho_{D}\left(M^{-1}\right) e^{\gamma}$. Then

$$
F_{K M}=\left.f\right|_{K M} \rho_{D}\left((K M)^{-1}\right) e^{\gamma}=\left.\chi_{\gamma}(K) f\right|_{M} \rho_{D}\left(M^{-1}\right) \rho_{D}\left(K^{-1}\right) e^{\gamma}=F_{M}
$$

for $K \in \Gamma_{1}(N)$. Hence $F=\sum_{M \in \Gamma_{1}(N) \backslash \Gamma} F_{M}$ is well defined. Now, let $K=$ $\left(\begin{array}{ll}a & b \\ c & d\end{array}\right) \in \Gamma$. Then

$$
\begin{aligned}
F(K \tau) & =\left.\sum_{M \in \Gamma_{1}(N) \backslash \Gamma} f\right|_{M}(K \tau) \rho_{D}\left(M^{-1}\right) e^{\gamma} \\
& =\left.(c \tau+d)^{k} \sum_{M \in \Gamma_{1}(N) \backslash \Gamma} f\right|_{M K}(\tau) \rho_{D}\left(M^{-1}\right) e^{\gamma} \\
& =\left.(c \tau+d)^{k} \rho_{D}(K) \sum_{M \in \Gamma_{1}(N) \backslash \Gamma} f\right|_{M K}(\tau) \rho_{D}\left(K^{-1}\right) \rho_{D}\left(M^{-1}\right) e^{\gamma} \\
& =\left.(c \tau+d)^{k} \rho_{D}(K) \sum_{M \in \Gamma_{1}(N) \backslash \Gamma} f\right|_{M K}(\tau) \rho_{D}\left((M K)^{-1}\right) e^{\gamma} \\
& =(c \tau+d)^{k} \rho_{D}(K) F(\tau)
\end{aligned}
$$

by shifting the summation index. Finally, the functions $F_{M}$ and $F$ are invariant under the stabilizer of $\gamma$ in $O(D)$ because the Weil representation commutes with $O(D)$.

The liftings satisfy some obvious relations. However, they are not equivalent. We will see that there are modular forms for the Weil representation which are not induced from $\Gamma_{0}(N)$. On the other hand, let $F=\sum_{\gamma \in D} F_{\gamma} e^{\gamma}$ be a modular form for $\rho_{D}$. Then

$$
\begin{aligned}
F & =\left.\frac{1}{\left|\Gamma / \Gamma_{1}(N)\right|} \sum_{M \in \Gamma_{1}(N) \backslash \Gamma} \sum_{\gamma \in D} F_{\gamma}\right|_{M} \rho_{D}\left(M^{-1}\right) e^{\gamma} \\
& =\frac{1}{\left|\Gamma / \Gamma_{1}(N)\right|} \sum_{\gamma \in D} F_{\Gamma_{1}(N), F_{\gamma}, \gamma} .
\end{aligned}
$$


Hence $F$ can be written as a linear combination of liftings from $\Gamma_{1}(N)$. Finally, let $V$ be the complex vector space generated by the components $F_{\gamma}$. Then $\Gamma$ acts on $V \otimes \mathbb{C}[D]$ by $\rho(M)\left(f \otimes e^{\gamma}\right)=\left.f\right|_{M^{-1}} \otimes \rho_{D}(M) e^{\gamma}$. The invariants $(V \otimes \mathbb{C}[D])^{\Gamma}$ are modular forms for $\rho_{D}$. This space is spanned by the functions $F_{\Gamma(N), F_{\gamma}, \mu}$.

We calculate the liftings explicitly.

The group $\Gamma_{0}(N)$ has index $N \prod_{p \mid N}(1+1 / p)$ in $\Gamma$ and $\sum_{c \mid N} \phi((c, N / c))$ classes of cusps. Let $a / c \in \mathbb{Q}$ with $(a, c)=1$. Then the equivalence class of $a / c$ as a cusp of $\Gamma_{0}(N)$ is determined by the invariants $(c, N)$ (a divisor of $N$ ) and $a c /(c, N)$ (a unit in $\mathbb{Z} /(c, N /(c, N)) \mathbb{Z}$ ). The width of $a / c$ is $t=$ $N /\left(c^{2}, N\right)$, and the stabilizer of $a / c$ in $\Gamma_{0}(N)$ is given by $\Gamma_{0}(N)_{a / c}=\left\{ \pm T_{a / c}^{n} \mid\right.$ $n \in \mathbb{Z}\}$ with

$$
T_{a / c}=M T^{t} M^{-1}=\left(\begin{array}{cc}
1-a c t & a^{2} t \\
-c^{2} t & 1+a c t
\end{array}\right)
$$

for any matrix $M \in \Gamma$ satisfying $M \infty=a / c$.

Let $M=\left(\begin{array}{ll}a & b \\ c & d\end{array}\right) \in \Gamma$. Then the cosets of $\Gamma_{0}(N) \backslash \Gamma$ sending $\infty$ to $a / c$ are given by $M T^{n}$, where $n$ ranges over a complete set of residues modulo $t=N /\left(c^{2}, N\right)$.

Let $D$ be a discriminant form of even signature and level dividing $N$, and let $f$ be a modular form on $\Gamma_{0}(N)$ with character $\chi_{D}$.

We denote the order of $\chi_{D}\left(T_{a / c}\right)$ by $m$.

For $M=\left(\begin{array}{ll}a & b \\ c & d\end{array}\right) \in \Gamma$, the function $\left.f\right|_{M}$ has a Fourier expansion of the form

$$
\left.f\right|_{M}(\tau)=\sum a_{n} q_{m t}^{n}
$$

with $q_{m t}=e(\tau / m t)$, and where $n$ is integral if $m=1$ and where $n$ is odd integral if $m=2$.

Let $a / c \in \mathbb{Q}$ with $(a, c)=1$, and let $\mu \in D^{c *}$. Then $\mu^{2} / 2=-n / m t \bmod 1$, where $n$ is integral if $m=1$ and where $n$ is odd integral if $m=2$.

Let $H$ be an isotropic subset of $D$ which is invariant under $(\mathbb{Z} / N \mathbb{Z})^{*}$. Then we can write $\sum_{\gamma \in H} e^{\gamma}$ as a linear combination of sums $\sum_{\gamma \in I} e^{\gamma}$, where $I$ is an isotropic subgroup of $D$. Therefore, it is sufficient to calculate the lift $F$ of $f$ on $H$ in the case that $H$ is an isotropic subgroup. We will assume this in the following.

We can write $F$ as

$$
F=\sum_{s \in \Gamma_{0}(N) \backslash P} F_{s},
$$


where $P$ is the set of cusps of $\Gamma$ and

$$
F_{s}=\left.\sum_{\substack{M \in \Gamma_{0}(N) \backslash \Gamma \\ M \infty=s}} \sum_{\gamma \in H} f\right|_{M} \rho_{D}\left(M^{-1}\right) e^{\gamma} .
$$

The function $F_{s}$ is $T$-invariant; that is, $F_{s}(T \tau)=\rho_{D}(T) F_{s}(\tau)$. We have the following.

TheOREM 3.2. Let $a / c \in \mathbb{Q}$ be a representative of $s$ with $(a, N)=1$ and $c \mid N$, and let $M=\left(\begin{array}{ll}a & b \\ c & d\end{array}\right) \in \Gamma$. Decompose $\left.f\right|_{M}=g_{m t, 0}+\cdots+g_{m t, m t-1}$ into $T$ invariant functions $g_{m t, j}$ satisfying $\left.g_{m t, j}\right|_{T}=e(j / m t) g_{m t, j}$, and for $w \in D^{c *}$ define $j_{w}$ by $w^{2} / 2=-j_{w} / m t \bmod 1$. Then

$$
\begin{aligned}
F_{s}= & \xi\left(M^{-1}\right) \frac{\sqrt{\left|D_{c}\right|}}{\sqrt{|D|}}\left|H \cap c H^{\perp}\right| \\
& \times \sum_{v \in H /\left(H \cap D^{c}\right)} \sum_{w \in\left(D^{c *} \cap D_{H}^{c *}\right)} e\left(d w_{c}^{2} / 2\right) \Phi_{H, a, c}(w) t g_{m t, j_{w}} e^{v+w},
\end{aligned}
$$

where

$$
\Phi_{H, a, c}(w)=\sum_{\gamma \in G_{H, c}} e\left(a c \gamma^{2} / 2+w \gamma\right)
$$

and

$$
G_{H, c}=\frac{c^{-1}(H) / D_{c}}{\left(\left(c^{-1}(H) \cap H^{\perp}\right)+D_{c}\right) / D_{c}} .
$$

The group $\Gamma_{1}(N)$ has index $N^{2} \prod_{p \mid N}\left(1-1 / p^{2}\right)$ in $\Gamma$ and

$$
\begin{array}{ll}
2 & \text { if } N=2, \\
3 & \text { if } N=4, \\
(1 / 2) \sum_{d \mid N} \phi(d) \phi(N / d) & \text { if } N=3 \text { or } N>4
\end{array}
$$

classes of cusps. Two cusps $a / c$ and $a^{\prime} / c^{\prime}$ in $\mathbb{Q}$ with $(a, c)=\left(a^{\prime}, c^{\prime}\right)=1$ are equivalent modulo $\Gamma_{1}(N)$ if and only if

$$
\left(\begin{array}{l}
a^{\prime} \\
c^{\prime}
\end{array}\right)= \pm\left(\begin{array}{c}
a+j c \\
c
\end{array}\right) \quad \bmod N
$$

for some $j$ (see [7, Section 3.8]). The cusp $1 / 2$ of $\Gamma_{1}(4)$ is irregular and has width $t=1$. All other classes of cusps $a / c$ are regular and have width $t=N /(c, N)$. For a cusp $a / c$ in $\mathbb{Q}$ with $(a, c)=1$ and width $t$, define as above 
$T_{a / c}=M T^{t} M^{-1}$, where $M$ is any matrix in $\Gamma$ sending $\infty$ to $a / c$. Then the stabilizer of $a / c$ in $\Gamma_{1}(N)$ is given by $\left\{T_{a / c}^{n} \mid n \in \mathbb{Z}\right\}$ if $a / c$ is regular and by $\left\{\left(-T_{a / c}\right)^{n} \mid n \in \mathbb{Z}\right\}$ if $a / c$ is irregular.

It is easy to prove the following result.

Proposition 3.3. Let $M=\left(\begin{array}{ll}a & b \\ c & d\end{array}\right) \in \Gamma$. Then the cosets of $\Gamma_{1}(N) \backslash \Gamma$ sending $\infty$ to a/c are represented by

$$
\begin{array}{ll}
M T^{n} & \text { if } N=2, \\
\pm M T^{n} & \text { if } N>2,
\end{array}
$$

where $n$ ranges over a complete set of residues modulo $t$.

Let $D$ be a discriminant form of even signature and level dividing $N$, and let $\gamma \in D$ with $\gamma^{2} / 2=-j / N \bmod 1$.

Proposition 3.4. Let $a / c \in \mathbb{Q}$ with $(a, c)=1$ be a cusp of $\Gamma_{1}(N)$. If $a / c$ is regular, then $\chi_{\gamma}\left(T_{a / c}\right)$ has order $m=(c, N) /(c, N, j)$. If a/c is irregular, then $\chi_{\gamma}\left(-T_{a / c}\right)$ has order $m=4 /(4, j)$.

Let $f$ be a modular form on $\Gamma_{1}(N)$ of weight $k$ and character $\chi_{\gamma}$.

Proposition 3.5. Let $a / c \in \mathbb{Q}$ with $(a, c)=1$ be a cusp of $\Gamma_{1}(N)$, and let $M=\left(\begin{array}{ll}a & b \\ c & d\end{array}\right) \in \Gamma$.

If $a / c$ is regular, then

$$
\left.f\right|_{M}(\tau)=\sum a_{n} q_{m t}^{n},
$$

where $n$ is integral and $n=a^{2} j m t / N$ mod $m$.

Suppose that a/c is irregular. If $k m$ is even, then

$$
\left.f\right|_{M}(\tau)=\sum a_{n} q_{m}^{n},
$$

where $n$ is integral and $n=k m / 2-j m / 4 \bmod m$. If $k m$ is odd, then

$$
\left.f\right|_{M}(\tau)=\sum a_{n} q_{2}^{n}
$$

with $n=1 \bmod 2$.

Proof. Let $a / c$ be regular. Then

$$
\left.f\right|_{M}\left(T^{t} \tau\right)=\left.f\right|_{M T^{t}}(\tau)=\left.f\right|_{T_{a / c} M}(\tau)=\left.\chi_{\gamma}\left(T_{a / c}\right) f\right|_{M}(\tau) .
$$


Since $\chi_{\gamma}\left(T_{a / c}\right)$ has order $m$, it follows that the function $\left.f\right|_{M}$ has an expansion in integral powers of $q_{m t}$. Write $\left.f\right|_{M}(\tau)=\sum a_{n} q_{m t}^{n}$. Then $\left.f\right|_{M}\left(T^{t} \tau\right)=$ $\sum a_{n} e(n t / m t) q_{m t}^{n}$ so that $f_{M}\left(T^{t} \tau\right)=\left.\chi_{\gamma}\left(T_{a / c}\right) f\right|_{M}(\tau)$ holds if and only if $e(n / m)=\chi_{\gamma}\left(T_{a / c}\right)$ for all $n$ with $a_{n} \neq 0$. But this is equivalent to $n=$ $a^{2} \mathrm{jmt} / N \bmod m$.

If $a / c$ is irregular, then

$$
\left.f\right|_{M}(T \tau)=\left.f\right|_{T_{a / c} M}(\tau)=\left.\chi_{\gamma}\left(-T_{a / c}\right) f\right|_{-M}(\tau)=\left.(-1)^{k} \chi_{\gamma}\left(-T_{a / c}\right) f\right|_{M}(\tau)
$$

and the statement follows by a similar argument.

Proposition 3.6. Let $a / c \in \mathbb{Q}$ with $(a, c)=1$ be a cusp of $\Gamma_{1}(N)$, and let $\mu \in a \gamma+D^{c *}$.

If $a / c$ is regular, then $\mu^{2} / 2=-n / m t \bmod 1$, where $n$ is an integer such that $n=a^{2} j m t / N$ mod $m$.

Suppose that $a / c$ is irregular and that $(-1)^{k} e(\operatorname{sign}(D) / 4) e(\gamma \mu)=1$. If $k m$ is even, then $\mu^{2} / 2=k / 2+j / 4 \bmod 1$. If $k m$ is odd, then $\mu^{2} / 2=1 / 2 \bmod 1$.

Proof. Choose a Jordan decomposition of $D$, and let $x_{c}$ be the canonical coset representative of $D^{c *}$. Write $\mu=a \gamma+\left(x_{c}+c \beta\right)$. Then

$$
\mu^{2} / 2=a^{2} \gamma^{2} / 2+x_{c}^{2} / 2+c^{2} \beta^{2} / 2+a c \gamma \beta+a x_{c} \gamma \bmod 1 .
$$

Let $a / c$ be regular. Then $t x_{c}^{2} / 2=t c^{2} \beta^{2} / 2=t a c \gamma \beta=\operatorname{tax}_{c} \gamma=0 \bmod 1$ and $m t a^{2} \gamma^{2} / 2=0 \bmod 1$. Therefore, we have $m t \mu^{2} / 2=0 \bmod 1$. Moreover, define $n=-m t \mu^{2} / 2 \bmod m$. Then $n=-m t a^{2} \gamma^{2} / 2=a^{2} j m t / N \bmod m$.

Now let $a / c$ be irregular. Then $N=4, a=1 \bmod 2$, and $c=2 \bmod 4$. The Jordan decomposition of $D$ is of the form $2_{I I}^{\epsilon_{2} n_{2}} 4_{I I}^{\epsilon_{4} n_{4}}$ or $2_{t_{2}}^{\epsilon_{2} n_{2}} 4_{I I}^{\epsilon_{4} n_{4}}$. In the first case, $\operatorname{sign}(D)=\operatorname{oddity}(D)=0 \bmod 4$ and $x_{c}=0$, and in the second case, $\operatorname{sign}(D)=\operatorname{oddity}(D)=t_{2} \bmod 4$ and $x_{c}^{2} / 2=t_{2} / 4=\operatorname{sign}(D) / 4 \bmod 1$. The formula for the norm of $\mu$ simplifies to

$$
\mu^{2} / 2=\gamma^{2} / 2+x_{c}^{2} / 2+c \gamma \beta+x_{c} \gamma \bmod 1 .
$$

Using $\gamma \mu=c \gamma^{2} / 2+x_{c} \gamma+c \gamma \beta \bmod 1$, we obtain

$$
\mu^{2} / 2=-\gamma^{2} / 2+x_{c}^{2} / 2+\gamma \mu \bmod 1 .
$$

Finally, $k / 2+\operatorname{sign}(D) / 4+\gamma \mu=k / 2+x_{c}^{2} / 2+\gamma \mu=0 \bmod 1$ so that

$$
\mu^{2} / 2=k / 2-\gamma^{2} / 2 \bmod 1 .
$$

This implies the last statement. 
Let $F$ be the lift of $f$ on $\gamma$. Then as above

$$
F=\sum_{s \in \Gamma_{1}(N) \backslash P} F_{s}
$$

with

$$
F_{s}=\left.\sum_{\substack{M \in \Gamma_{1}(N) \backslash \Gamma \\ M \infty=s}} f\right|_{M} \rho_{D}\left(M^{-1}\right) e^{\gamma}
$$

The functions $F_{s}$ are given in the following theorem.

THEOREM 3.7. Let $a / c \in \mathbb{Q}$ with $(a, c)=1$ be a representative of $s$, and let $M=\left(\begin{array}{ll}a & b \\ c & d\end{array}\right) \in \Gamma$.

If $s$ is regular here, then write $\left.f\right|_{M}=g_{m t, 0}+\cdots+g_{m t, m t-1}$ with $\left.g_{m t, j}\right|_{T}=$ $e(j / m t) g_{m t, j}$, and for $\mu \in a \gamma+D^{c *}$, define $j_{\mu}$ by $\mu^{2} / 2=-j_{\mu} / m t \bmod 1$. Then

$$
\begin{aligned}
F_{s}= & \xi\left(M^{-1}\right) \frac{\sqrt{\left|D_{c}\right|}}{\sqrt{|D|}} \sum_{\mu \in a \gamma+D^{c *}} e\left(d(\mu-a \gamma)_{c}^{2} / 2\right) e(b \mu \gamma) e\left(-a b \gamma^{2} / 2\right) \\
& \times t g_{m t, j_{\mu}}\left\{e^{\mu}+(-1)^{k} e(\operatorname{sign}(D) / 4) e^{-\mu}\right\}
\end{aligned}
$$

if $N>2$, and

$$
F_{s}=\xi\left(M^{-1}\right) \frac{\sqrt{\left|D_{c}\right|}}{\sqrt{|D|}} \sum_{\mu \in a \gamma+D^{c *}} e\left(d(\mu-a \gamma)_{c}^{2} / 2\right) e(b \gamma \mu) e\left(-a b \gamma^{2} / 2\right) t g_{m t, j_{\mu}} e^{\mu}
$$

if $N=2$.

If $a / c$ is irregular, then

$$
\begin{aligned}
F_{s}= & \xi\left(M^{-1}\right) \frac{\sqrt{\left|D_{c}\right|}}{\sqrt{|D|}} \sum_{\mu \in a \gamma+D^{c *}} e\left(d(\mu-a \gamma)_{c}^{2} / 2\right) e(b \mu \gamma) e\left(-a b \gamma^{2} / 2\right) \\
& \times\left.\left\{1+(-1)^{k} e(\operatorname{sign}(D) / 4) e(\mu \gamma)\right\} f\right|_{M} e^{\mu}
\end{aligned}
$$

Proof. Let $s$ be regular, and let $N>2$. The cosets of $\Gamma_{1}(N)$ sending $\infty$ to $s$ are represented by $\pm M T^{n}$, where $n$ ranges over a complete set of residues modulo $t=N /(c, N)$. Using $\left.f\right|_{-1}=(-1)^{k} f$ and $\rho_{D}(-1) e^{\gamma}=$ $e(\operatorname{sign}(D) / 4) e^{-\gamma}$, we get 


$$
\begin{aligned}
F_{s}= & \left.\sum_{n \bmod t} f\right|_{M T^{n}} \rho_{D}\left(T^{-n}\right) \rho_{D}\left(M^{-1}\right) e^{\gamma} \\
& +\left.(-1)^{k} e(\operatorname{sign}(D) / 4) \sum_{n \bmod t} f\right|_{M T^{n}} \rho_{D}\left(T^{-n}\right) \rho_{D}\left(M^{-1}\right) e^{-\gamma} .
\end{aligned}
$$

Now

$$
\rho_{D}\left(M^{-1}\right) e^{\gamma}=\xi\left(M^{-1}\right) \frac{\sqrt{\left|D_{c}\right|}}{\sqrt{|D|}} \sum_{\beta \in D^{c *}} e\left(d \beta_{c}^{2} / 2\right) e(b \beta \gamma) e\left(a b \gamma^{2} / 2\right) e^{a \gamma+\beta}
$$

so that

$$
\begin{aligned}
F_{s}= & \left.\xi\left(M^{-1}\right) \frac{\sqrt{\left|D_{c}\right|}}{\sqrt{|D|}} \sum_{n \bmod t} f\right|_{M T^{n}} \rho_{D}\left(T^{-n}\right) \\
& \times\left\{\sum_{\beta \in D^{c *}} e\left(d \beta_{c}^{2} / 2\right) e(b \beta \gamma) e\left(a b \gamma^{2} / 2\right) e^{a \gamma+\beta}\right. \\
& \left.+(-1)^{k} e(\operatorname{sign}(D) / 4) \sum_{\beta \in D^{c *}} e\left(d \beta_{c}^{2} / 2\right) e(-b \beta \gamma) e\left(a b \gamma^{2} / 2\right) e^{-a \gamma+\beta}\right\} .
\end{aligned}
$$

Replacing $\beta$ by $-\beta$ in the last sum and putting $\mu=a \gamma+\beta$ gives

$$
\begin{aligned}
F_{s}= & \left.\xi\left(M^{-1}\right) \frac{\sqrt{\left|D_{c}\right|}}{\sqrt{|D|}} \sum_{\mu \in a \gamma+D^{c *}} \sum_{n \bmod t} f\right|_{M T^{n}} e\left(n \mu^{2} / 2\right) \\
& \times e\left(d(\mu-a \gamma)_{c}^{2} / 2\right) e(b \mu \gamma) e\left(-a b \gamma^{2} / 2\right)\left\{e^{\mu}+(-1)^{k} e(\operatorname{sign}(D) / 4) e^{-\mu}\right\}
\end{aligned}
$$

The formula now follows from

$$
\left.\sum_{n \bmod t} f\right|_{M T^{n}} e\left(n \mu^{2} / 2\right)=\operatorname{tg}_{m t, j_{\mu}} .
$$

The case $N=2$ is now clear.

Suppose that $s$ is irregular; that is, $N=4, a=1 \bmod 2$, and $c=2 \bmod 4$. We argue slightly differently here because $a \gamma+D^{c *}=-a \gamma-D^{c *}$ in this case. We have

$$
\begin{aligned}
F_{s} & =\left.f\right|_{M} \rho_{D}\left(M^{-1}\right) e^{\gamma}+\left.f\right|_{-M} \rho_{D}\left(-M^{-1}\right) e^{-\gamma} \\
& =\left.f\right|_{M} \rho_{D}\left(M^{-1}\right) e^{\gamma}+\left.(-1)^{k} e(\operatorname{sign}(D) / 4) f\right|_{M} \rho_{D}\left(M^{-1}\right) e^{-\gamma}
\end{aligned}
$$

and 


$$
\begin{aligned}
& \rho_{D}\left(M^{-1}\right) e^{-\gamma} \\
& =\xi\left(M^{-1}\right) \frac{\sqrt{\left|D_{c}\right|}}{\sqrt{|D|}} \sum_{\beta \in D^{c *}} e\left(d \beta_{c}^{2} / 2\right) e(-b \beta \gamma) e\left(a b \gamma^{2} / 2\right) e^{-a \gamma+\beta} \\
& =\xi\left(M^{-1}\right) \frac{\sqrt{\left|D_{c}\right|}}{\sqrt{|D|}} \sum_{\beta \in 2 a \gamma+D^{c *}} e\left(d \beta_{c}^{2} / 2\right) e(-b \beta \gamma) e\left(a b \gamma^{2} / 2\right) e^{-a \gamma+\beta} \\
& =\xi\left(M^{-1}\right) \frac{\sqrt{\left|D_{c}\right|}}{\sqrt{|D|}} \sum_{\beta \in D^{c *}} e\left(d(2 a \gamma+\beta)_{c}^{2} / 2\right) e(-b(2 a \gamma+\beta) \gamma) e\left(a b \gamma^{2} / 2\right) e^{a \gamma+\beta} \\
& =\xi\left(M^{-1}\right) \frac{\sqrt{\left|D_{c}\right|}}{\sqrt{|D|}} \sum_{\beta \in D^{c *}} e\left(d \beta_{c}^{2} / 2\right) e\left(c \gamma^{2} / 2+\gamma \beta\right) e(b \gamma \beta) e\left(a b \gamma^{2} / 2\right) e^{a \gamma+\beta}
\end{aligned}
$$

because $d(2 a \gamma+\beta)_{c}^{2} / 2=d \beta_{c}^{2} / 2+c \gamma^{2} / 2+\gamma \beta \bmod 1$ and $-b(2 a \gamma+\beta) \gamma=$ $b \beta \gamma \bmod 1$. Hence

$$
\begin{aligned}
F_{s}= & \xi\left(M^{-1}\right) \frac{\sqrt{\left|D_{c}\right|}}{\sqrt{|D|}} \sum_{\beta \in D^{c *}} e\left(d \beta_{c}^{2} / 2\right) e(b \gamma \beta) e\left(a b \gamma^{2} / 2\right) \\
& \times\left.\left\{1+(-1)^{k} e(\operatorname{sign}(D) / 4) e\left(c \gamma^{2} / 2+\gamma \beta\right)\right\} f\right|_{M} e^{a \gamma+\beta} .
\end{aligned}
$$

Let $\mu=a \gamma+\beta$. Then $a b \gamma^{2} / 2+b \gamma \beta=-a b \gamma^{2} / 2+b \gamma \mu \bmod 1$ and $c \gamma^{2} / 2+$ $\gamma \beta=\gamma \mu \bmod 1$ so that

$$
\begin{aligned}
F_{s}= & \xi\left(M^{-1}\right) \frac{\sqrt{\left|D_{c}\right|}}{\sqrt{|D|}} \sum_{\mu \in a \gamma+D^{c *}} e\left(d(\mu-a \gamma)_{c}^{2} / 2\right) e(b \mu \gamma) e\left(-a b \gamma^{2} / 2\right) \\
& \times\left.\left\{1+(-1)^{k} e(\operatorname{sign}(D) / 4) e(\mu \gamma)\right\} f\right|_{M} e^{\mu}
\end{aligned}
$$

This proves the theorem.

The group $\Gamma(N)$ has index $N^{3} \prod_{p \mid N}\left(1-1 / p^{2}\right)$ in $\Gamma$ and

$$
\begin{array}{ll}
3 & \text { if } N=2, \\
\left(N^{2} / 2\right) \prod_{p \mid N}\left(1-1 / p^{2}\right) & \text { if } N>2
\end{array}
$$

classes of cusps. Two cusps $a / c$ and $a^{\prime} / c^{\prime}$ in $\mathbb{Q}$ with $(a, c)=\left(a^{\prime}, c^{\prime}\right)=1$ are equivalent modulo $\Gamma(N)$ if and only if

$$
\left(\begin{array}{l}
a^{\prime} \\
c^{\prime}
\end{array}\right)= \pm\left(\begin{array}{l}
a \\
c
\end{array}\right) \quad \bmod N
$$


The width of $a / c$ is $N$, and the stabilizer of $a / c$ in $\Gamma(N)$ is generated by $T_{a / c}$ defined as above.

Let $M=\left(\begin{array}{ll}a & b \\ c & d\end{array}\right) \in \Gamma$. Then the cosets of $\Gamma(N) \backslash \Gamma$ sending $\infty$ to $a / c$ are represented by

$$
\begin{array}{ll}
M T^{n} & \text { if } N=2, \\
\pm M T^{n} & \text { if } N>2,
\end{array}
$$

where $n$ ranges over a complete set of residues modulo $N$.

Let $D$ be a discriminant form of even signature and level dividing $N$, and let $f$ be a modular form on $\Gamma(N)$ of weight $k$.

The function $\left.f\right|_{M}$ has a Fourier expansion in integral powers of $q_{N}$ for all $M \in \Gamma$.

Let $F$ be the lift of $f$ on $\gamma \in D$. Then $F=\sum_{s \in \Gamma(N) \backslash P} F_{s}$, and as above we find the following.

TheOREM 3.8. Let $a / c \in \mathbb{Q}$ with $(a, c)=1$ be a representative of $s$, and let $M=\left(\begin{array}{ll}a & b \\ c & d\end{array}\right) \in \Gamma$. Decompose $\left.f\right|_{M}$ into T-eigenfunctions $g_{N, j}$, and for $\mu \in$ $a \gamma+D^{c *}$, define $j_{\mu}$ by $\mu^{2} / 2=-j_{\mu} / N \bmod 1$. Then

$$
\begin{aligned}
F_{s}= & \xi\left(M^{-1}\right) \frac{\sqrt{\left|D_{c}\right|}}{\sqrt{|D|}} \sum_{\mu \in a \gamma+D^{c *}} e\left(d(\mu-a \gamma)_{c}^{2} / 2\right) e(b \mu \gamma) e\left(-a b \gamma^{2} / 2\right) \\
& \times N g_{N, j_{\mu}}\left\{e^{\mu}+(-1)^{k} e(\operatorname{sign}(D) / 4) e^{-\mu}\right\}
\end{aligned}
$$

if $N>2$, and

$$
F_{s}=\xi\left(M^{-1}\right) \frac{\sqrt{\left|D_{c}\right|}}{\sqrt{|D|}} \sum_{\mu \in a \gamma+D^{c *}} e\left(d(\mu-a \gamma)_{c}^{2} / 2\right) e(b \gamma \mu) e\left(-a b \gamma^{2} / 2\right) N g_{N, j_{\mu}} e^{\mu}
$$

if $N=2$.

\section{$\S 4$. Induction from isotropic subgroups}

Let $L$ be an even lattice, and let $M$ be a sublattice of $L$ of finite index. We show that a modular form on the discriminant form of $L$ induces in a canonical way a modular form on the discriminant form of $M$.

Let $D$ be a discriminant form of even signature. Let $H$ be an isotropic subgroup of $D$, and let $H^{\perp}$ be the orthogonal complement of $H$ in $D$. Then $D_{H}=H^{\perp} / H$ is a discriminant form of the same signature as $D$ with 
$\left|D_{H}\right|=|D| /|H|^{2}$. Let $F_{D_{H}}=\sum_{\gamma \in D_{H}} F_{D_{H}, \gamma} e^{\gamma}$ be a modular form for $\rho_{D_{H}}$. Define

$$
F=\sum_{\gamma \in H^{\perp}} F_{D_{H}, \gamma+H} e^{\gamma} .
$$

Then we have the following.

THEOREM 4.1. The function $F$ is a modular form for $\rho_{D}$.

Proof. It is sufficient to show that $F=\sum_{\gamma \in D} F_{\gamma} e^{\gamma}$ transforms correctly under the generators of $\Gamma$. This is clear for $T$.

For $\gamma \in H^{\perp}$, we have

$$
\begin{aligned}
\left.F_{\gamma}\right|_{S}(\tau) & =\left.F_{D_{H}, \gamma+H}\right|_{S}(\tau) \\
& =\frac{e\left(\operatorname{sign}\left(D_{H}\right) / 8\right)}{\sqrt{\left|D_{H}\right|}} \sum_{\beta \in D_{H}} e((\gamma+H) \beta) F_{D_{H}, \beta}(\tau) \\
& =\frac{e\left(\operatorname{sign}\left(D_{H}\right) / 8\right)}{|H| \sqrt{\left|D_{H}\right|}}|H| \sum_{\beta \in D_{H}} e((\gamma+H) \beta) F_{D_{H}, \beta}(\tau) \\
& =\frac{e\left(\operatorname{sign}\left(D_{H}\right) / 8\right)}{|H| \sqrt{\left|D_{H}\right|}} \sum_{\beta \in H^{\perp}} e(\gamma \beta) F_{\beta}(\tau) \\
& =\frac{e(\operatorname{sign}(D) / 8)}{\sqrt{|D|}} \sum_{\beta \in D} e(\gamma \beta) F_{\beta}(\tau) .
\end{aligned}
$$

If $\gamma \notin H^{\perp}$, then $\left.F_{\gamma}\right|_{S}(\tau)=0$ and

$$
\begin{aligned}
\sum_{\beta \in D} e(\gamma \beta) F_{\beta}(\tau) & =\sum_{\beta \in H^{\perp}} e(\gamma \beta) F_{\beta}(\tau) \\
& =\sum_{\beta \in H^{\perp} / H} \sum_{\mu \in H} e(\gamma(\beta+\mu)) F_{\beta+\mu}(\tau) \\
& =\sum_{\beta \in D_{H}} e((\gamma+H) \beta) F_{D_{H}, \beta}(\tau) \sum_{\mu \in H} e(\gamma \mu) \\
& =0
\end{aligned}
$$

because $\sum_{\mu \in H} e(\gamma \mu)$ is the sum over a nontrivial character of $H$ and therefore is 0 .

We can easily construct some automorphisms of $F$. An automorphism of $D$ stabilizes $H$ as a set if and only if it stabilizes $H^{\perp}$. Such automorphisms act on $H^{\perp} / H=D_{H}$ so that we get a natural map from the stabilizer of 
$H$ in $O(D)$ to $O\left(D_{H}\right)$. The inverse image of $O\left(F_{D_{H}}\right)$ under this map is a subgroup of $O(F)$.

A standard situation in which the above result can be applied is the following. Let $L$ be an even lattice of even signature, and let $M$ be a sublattice of $L$ of finite index. Then we have embeddings $M \subset L \subset L^{\prime} \subset M^{\prime}$, and $H=L / M$ is an isotropic subgroup of $D_{M}=M^{\prime} / M$. The orthogonal complement of $H$ in $D_{M}$ is $H^{\perp}=L^{\prime} / M$, and $H^{\perp} / H$ is naturally isomorphic to $D_{L}=L^{\prime} / L$. The theorem then shows that a modular form for the discriminant form of $L$ induces a modular form for the discriminant form of the sublattice $M$.

\section{$\S 5$. Discriminant forms of square-free level}

Let $D$ be a discriminant form of even signature and level $N$. Let $f$ be a modular form on $\Gamma_{0}(N)$ of character $\chi_{D}$. Then $F_{\Gamma_{0}(N), f, 0}$ is a modular form for $\rho_{D}$ which is invariant under $O(D)$. We show now that every modular form for $\rho_{D}$ which is invariant under $O(D)$ can be obtained in this way if $N$ is square-free.

First, we describe some properties of discriminant forms of prime level.

Proposition 5.1. Let $D$ be a discriminant form of prime level, and let $\beta$ and $\gamma$ be two nonzero elements in $D$ of the same norm. Then $\beta$ and $\gamma$ are conjugate under $O(D)$.

Proof. Let $p$ be the level of $D$. If $p$ is an odd prime, we can consider $D$ as a vector space over $\mathbb{F}_{p}$ with a quadratic form. The statement then follows from Witt's theorem (see [10]). For $p=2$, we can argue as follows. The statement is true if $D$ has 2-rank 2. Suppose that the 2-rank is at least 4 . If $\beta^{2} / 2=$ $0 \bmod 1$, then there is an $\alpha \in D$ such that $\alpha \beta=1 / 2 \bmod 1$. Replacing $\alpha$ by $\alpha+\beta$ if necessary, we can assume that $\alpha^{2} / 2=0 \bmod 1$. Similarly, if $\beta^{2} / 2=1 / 2 \bmod 1$, we can find $\alpha \in D$ such that $\alpha \beta=\alpha^{2} / 2=1 / 2 \bmod 1$. In both cases, $D$ decomposes into the orthogonal sum $D=\langle\alpha, \beta\rangle \oplus\langle\alpha, \beta\rangle^{\perp}$. This proves the proposition for $p=2$.

For a discriminant form $D$, we denote by $I$ the subset of isotropic elements and we denote by $I_{k}$ the isotropic elements of order $k$.

Proposition 5.2. Let $D$ be a discriminant form of prime level $p$. Then we have, for $\gamma \in I_{p}$,

$$
\sum_{\beta \in I_{p}} e(\beta \gamma)=\left|I_{p}\right|-\frac{|D|}{p}
$$


Proof. We prove the statement in the case that $D$ has Jordan decomposition $p^{\epsilon n}$ with $p$ odd and $n \geq 3$. The other cases are similar. We can assume that

$$
D=D^{\prime} \oplus D^{\prime \prime}
$$

where $D^{\prime}$ is generated by $\gamma_{1}, \gamma_{2}$ with $\gamma_{1}^{2} / 2=1 / p \bmod 1, \gamma_{2}^{2} / 2=-1 / p \bmod 1$, $\gamma_{1} \gamma_{2}=0$ (it has Jordan decomposition $p^{\epsilon^{\prime} 2}$ with $\epsilon^{\prime}=\left(\frac{2}{p}\right)\left(\frac{-2}{p}\right)=\left(\frac{-1}{p}\right)$ and contains $\gamma$ ) and where $D^{\prime \prime}$ has Jordan decomposition $p^{\epsilon^{\prime \prime}(n-2)}$ with $\epsilon^{\prime \prime}=$ $\left(\frac{-1}{p}\right) \epsilon$. Then

$$
\gamma^{\perp}=\langle\gamma\rangle \oplus D^{\prime \prime}
$$

so that by [12, Proposition 3.2],

$$
\begin{aligned}
\left|\gamma^{\perp} \cap I\right| & =p \begin{cases}p^{n-3}+\epsilon^{\prime \prime}\left(\frac{-1}{p}\right)^{(n-2) / 2}\left(p^{(n-2) / 2}-p^{(n-4) / 2}\right) & \text { if } n \text { is even, } \\
p^{n-3} & \text { if } n \text { is odd }\end{cases} \\
& = \begin{cases}p^{n-2}+\epsilon\left(\frac{-1}{p}\right)^{n / 2}\left(p^{n / 2}-p^{(n-2) / 2}\right) & \text { if } n \text { is even, } \\
p^{n-2} & \text { if } n \text { is odd. }\end{cases}
\end{aligned}
$$

Define $A_{j}=\{\beta \in I \mid \gamma \beta=j / p \bmod 1\}$. Then

$$
I=\bigcup_{j=0}^{p-1} A_{j} .
$$

We have $A_{0}=\gamma^{\perp} \cap I$ and $\left|A_{1}\right|=\cdots=\left|A_{p-1}\right|=m$ because multiplication by elements in $(\mathbb{Z} / p Z)^{*}$ maps the sets into each other. Hence applying again [12, Proposition 3.2], we obtain

$$
m=\frac{|I|-\left|A_{0}\right|}{p-1}=p^{n-2} .
$$

It follows that

$$
\begin{aligned}
\sum_{\beta \in I} e(\gamma \beta) & =\left|A_{0}\right|+m \sum_{j=1}^{p-1} e(j / p) \\
& =\left|A_{0}\right|-m \\
& =\left|A_{0}\right|-p^{n-2} \\
& =|I|-p^{n-1} \\
& =\left|I_{p}\right|+1-\frac{|D|}{p} .
\end{aligned}
$$

This proves the statement. 
Let $D$ be a discriminant form of square-free level $N$. Then Proposition 5.1 implies that two elements of $D$ are in the same orbit under $O(D)$ if and only if they have the same norm and order. Let $F=\sum_{\gamma \in D} F_{\gamma} e^{\gamma}$ be a modular form for $\rho_{D}$ which is invariant under $O(D)$. Then the components $F_{\gamma}$ with isotropic $\gamma$ are modular forms on $\Gamma_{0}(N)$ with character $\chi_{D}$.

Proposition 5.3. Let $D$ be a discriminant form of square-free level $N$, and let $F=\sum_{\gamma \in D} F_{\gamma} e^{\gamma}$ be a modular form for $\rho_{D}$ which is invariant under $O(D)$. Then the complex vector space $W$ spanned by the components $F_{\gamma}$, $\gamma \in D$ is generated by the functions $\left.F_{0}\right|_{M}, M \in \Gamma$. In particular, $F=0$ if $F_{0}=0$.

Proof. Clearly $W$ contains the functions $\left.F_{0}\right|_{M}, M \in \Gamma$. Let $M=\left(\begin{array}{ll}a & b \\ c & d\end{array}\right) \in \Gamma$. Then $\left.F_{0}\right|_{M}$ decomposes into $T$-eigenfunctions, that is,

$$
\left.F_{0}\right|_{M}=g_{t, 0}+\cdots+g_{t, t-1}
$$

where $t=N /\left(c^{2}, N\right)$ is the width of $a / c$ and $\left.g_{t, j}\right|_{T}=e(j / t) g_{t, j}$. The function $g_{t, j}$ can be written as

$$
g_{t, j}=\left.\frac{1}{t} \sum_{n \bmod t} e(-j n / t) F_{0}\right|_{M T^{n}}
$$

and hence it is in $W$. Now let $M=\left(\begin{array}{ll}a & b \\ c & d\end{array}\right) \in \Gamma$ with $c \mid N$ and let $d=0 \bmod c^{\prime}$, where $c^{\prime}=N / c$. Then

$$
\left.F_{0}\right|_{M}=\bar{\xi}\left(M^{-1}\right) \frac{1}{\sqrt{\left|D_{c^{\prime}}\right|}} \sum_{\gamma \in D_{c^{\prime}}} F_{\gamma}
$$

Since $F_{\gamma}$ is determined by the norm and order of $\gamma$, we see that $F_{\gamma}$ can be written as a linear combination of functions $\left.F_{0}\right|_{M}, M \in \Gamma$.

The main result of this section is the following.

THEOREM 5.4. Let $D$ be a discriminant form of square-free level $N$, and let $F=\sum_{\gamma \in D} F_{\gamma} e^{\gamma}$ be a modular form for $\rho_{D}$ which is invariant under $O(D)$. Let $N_{R}$ be the product over the primes with nonvanishing $I_{p}$. For $k \mid N_{R}$, define $F_{k}=F_{\gamma}$, where $\gamma$ is any element in $I_{k}$. Then the functions $F_{k}$ span the subspace $W_{0}$ of $W$ with $T$-eigenvalue e(0). Define 


$$
\begin{aligned}
\Phi: W_{0} & \longrightarrow W_{0}, \\
f & \longmapsto 0 \text {-component of } F_{\Gamma_{0}(N), f, 0} .
\end{aligned}
$$

Then

$$
\Phi\left(F_{k}\right)=\sum_{j \mid N_{R}} a_{j k} F_{j}
$$

with

$$
\begin{aligned}
a_{j k} & =\frac{N}{|D|}\left|I_{j}\right| \sum_{c \mid(N / j, N / k)} \frac{\left|D_{c}\right|}{c} \\
& =\frac{N}{|D|} \prod_{p \mid j}\left|I_{p}\right| \prod_{p \mid(N / j, N / k)}\left(1+\frac{\left|D_{p}\right|}{p}\right) .
\end{aligned}
$$

The matrix $A=\left(a_{j k}\right)$ has determinant

$$
\operatorname{det}(A)=\left(\frac{N}{|D|}\right)^{\sigma\left(N_{R}\right)}\left(\sum_{d \mid N / N_{R}} \frac{\left|D_{d}\right|}{d}\right)^{\sigma\left(N_{R}\right)} \prod_{d \mid N_{R}}\left|I_{d}\right| \prod_{d \mid N_{R}} \frac{\left|D_{d}\right|}{d}
$$

In particular, $\Phi$ is invertible.

Proof. Let $\gamma \in I_{k}$, where $k \mid N_{R}$. Then

$$
\Phi\left(F_{\gamma}\right)=\sum_{s \in \Gamma_{0}(N) \backslash P} \Phi_{s}\left(F_{\gamma}\right)
$$

with

$$
\Phi_{s}\left(F_{\gamma}\right)=\left.\sum_{\substack{M \in \Gamma_{0}(N) \backslash \Gamma \\ M \infty=s}} F_{\gamma}\right|_{M}\left\langle\rho_{D}\left(M^{-1}\right) e^{0}, e^{0}\right\rangle .
$$

The cusps of $\Gamma_{0}(N)$ are given by $1 / c$, where $c$ ranges over the positive divisors of $N$, and the cusp $1 / c$ has width $c^{\prime}=N / c$.

Let $c \mid N$. Choose $M=\left(\begin{array}{ll}a & b \\ c & d\end{array}\right) \in \Gamma$ with $d=1 \bmod c$ and $d=0 \bmod c^{\prime}$. Then

$$
\begin{aligned}
\Phi_{1 / c}\left(F_{\gamma}\right) & =\left.\sum_{n \bmod c^{\prime}} F_{\gamma}\right|_{M T^{n}}\left\langle\rho_{D}\left(\left(M T^{n}\right)^{-1}\right) e^{0}, e^{0}\right\rangle \\
& =\sum_{n \bmod c^{\prime}} \sum_{\alpha \in D} F_{\alpha}\left\langle\rho_{D}\left(M T^{n}\right) e^{\alpha}, e^{\gamma}\right\rangle\left\langle\rho_{D}\left(\left(M T^{n}\right)^{-1}\right) e^{0}, e^{0}\right\rangle .
\end{aligned}
$$


The function $\Phi_{1 / c}\left(F_{\gamma}\right)$ is $T$-invariant so that the contributions of the $\alpha \in D$ with nonzero norm cancel each other. Hence

$$
\Phi_{1 / c}\left(F_{\gamma}\right)=\frac{N}{c} \sum_{\substack{\alpha \in D \\ \alpha^{2} / 2=0 \bmod 1}} F_{\alpha}\left\langle e^{\alpha}, \rho_{D}\left(M^{-1}\right) e^{\gamma}\right\rangle\left\langle\rho_{D}\left(M^{-1}\right) e^{0}, e^{0}\right\rangle .
$$

Now

$$
\begin{aligned}
\rho_{D}\left(M^{-1}\right) e^{\gamma} & =\xi\left(M^{-1}\right) \frac{\sqrt{\left|D_{c}\right|}}{\sqrt{|D|}} \sum_{\beta \in D_{c^{\prime}}} e(b \beta \gamma) e^{a \gamma+\beta} \\
& =\xi\left(M^{-1}\right) \frac{\sqrt{\left|D_{c}\right|}}{\sqrt{|D|}} \sum_{\beta \in D_{c^{\prime}}} e\left(-c^{-1} \beta \gamma\right) e^{a \gamma+\beta}
\end{aligned}
$$

where $c^{-1}$ is the inverse of $c$ modulo $c^{\prime}$ so that

$$
\begin{aligned}
\Phi_{1 / c}\left(F_{\gamma}\right) & =\frac{N}{c} \frac{\left|D_{c}\right|}{|D|} \sum_{\substack{\alpha \in D \\
\alpha^{2} / 2=0 \bmod 1}} F_{\alpha} \sum_{\beta \in D_{c^{\prime}}} e\left(c^{-1} \beta \gamma\right)\left\langle e^{\alpha}, e^{a \gamma+\beta}\right\rangle \\
& =\frac{N}{c} \frac{\left|D_{c}\right|}{|D|} \sum_{\substack{\alpha \in\left(\gamma+D_{c^{\prime}}\right) \\
\alpha^{2} / 2=0 \bmod 1}} e\left(c^{-1} \alpha \gamma\right) F_{\alpha}
\end{aligned}
$$

and

$$
\Phi\left(F_{\gamma}\right)=\sum_{c \mid N} \frac{N}{c} \frac{\left|D_{c}\right|}{|D|} \sum_{\substack{\alpha \in\left(\gamma+D_{c^{\prime}}\right) \\ \alpha^{2} / 2=0 \bmod 1}} e\left(c^{-1} \alpha \gamma\right) F_{\alpha} .
$$

Since

$$
\sum_{\substack{\alpha \in\left(\gamma+D_{c^{\prime}}\right) \\ \alpha^{2} / 2=0 \bmod 1}} e\left(c^{-1} \alpha \gamma\right) F_{\alpha}=\sum_{j \mid N_{R}} F_{j} \sum_{\alpha \in\left(\gamma+D_{c^{\prime}}\right) \cap I_{j}} e\left(c^{-1} \alpha \gamma\right)
$$

we get

$$
\Phi\left(F_{k}\right)=\sum_{j \mid N_{R}} a_{j k} F_{j}
$$

with

$$
a_{j k}=\sum_{c \mid N} \frac{N}{c} \frac{\left|D_{c}\right|}{|D|} \sum_{\alpha \in\left(\gamma+D_{c^{\prime}}\right) \cap I_{j}} e\left(c^{-1} \alpha \gamma\right)
$$


The elements in $\gamma+D_{c^{\prime}}$ have order $(k, c) d$, where $d \mid c^{\prime}$ and the elements in $I_{j}$ have order $j$. Hence

$$
\begin{aligned}
a_{j k}= & \sum_{\substack{c|N \\
(k, c)| j \mid(k, c) c^{\prime}}} \frac{N}{c} \frac{\left|D_{c}\right|}{|D|} \sum_{\alpha \in\left(\gamma+D_{c^{\prime}}\right) \cap I_{j}} e\left(c^{-1} \alpha \gamma\right) \\
= & \sum_{\substack{c|N \\
(k, c)| j \mid(k, c) c^{\prime}}} \frac{N}{c} \frac{\left|D_{c}\right|}{|D|} \sum_{\alpha \in I_{j /(k, c)}} e\left(c^{-1} \alpha \gamma\right) \\
= & \sum_{\substack{c \mid N \\
(j, c)=(k, c)}} \frac{N}{c} \frac{\left|D_{c}\right|}{|D|} \sum_{\alpha \in I_{\left(j, c^{\prime}\right)}} e(\alpha \gamma) .
\end{aligned}
$$

We can evaluate the last sum using Proposition 5.2. We get

$$
a_{j k}=\frac{N}{|D|} \sum_{\substack{c \mid N \\(j, c)=(k, c)}} \frac{\left|D_{c}\right|}{c} \prod_{\substack{p \mid\left(j, c^{\prime}\right) \\ p \nmid k}}\left|I_{p}\right| \prod_{\substack{p\left|\left(j, c^{\prime}\right) \\ p\right| k}}\left(\left|I_{p}\right|-\frac{\left|D_{p}\right|}{p}\right) .
$$

Induction on the number of prime divisors of $N$ shows

$$
\begin{aligned}
a_{j k} & =\frac{N}{|D|} \prod_{p \mid j}\left|I_{p}\right| \sum_{c \mid(N / j, N / k)} \frac{\left|D_{c}\right|}{c} \\
& =\frac{N}{|D|}\left|I_{j}\right| \prod_{p \mid(N / j, N / k)}\left(1+\frac{\left|D_{p}\right|}{p}\right) \\
& =\frac{N}{|D|} \prod_{p \mid N / N_{R}}\left(1+\frac{\left|D_{p}\right|}{p}\right)\left|I_{j}\right| \prod_{p \mid\left(N_{R} / j, N_{R} / k\right)}\left(1+\frac{\left|D_{p}\right|}{p}\right) .
\end{aligned}
$$

We can write $A=\left(a_{j k}\right)$ as

$$
A=\frac{N}{|D|} \prod_{p \mid N / N_{R}}\left(1+\frac{\left|D_{p}\right|}{p}\right) B C,
$$

where $B=\left(b_{j k}\right)$ is a diagonal matrix with entries

$$
b_{j k}=\delta_{j k}\left|I_{j}\right|
$$


and $C=\left(c_{j k}\right)$ is a symmetric matrix with entries

$$
c_{j k}=\prod_{p \mid\left(N_{R} / j, N_{R} / k\right)}\left(1+\frac{\left|D_{p}\right|}{p}\right) .
$$

We have

$$
\operatorname{det}(B)=\prod_{d \mid N_{R}}\left|I_{d}\right|
$$

and

$$
\operatorname{det}(C)=\prod_{d \mid N_{R}} \frac{\left|D_{d}\right|}{d}
$$

so that

$$
\operatorname{det}(A)=\left(\frac{N}{|D|}\right)^{\sigma\left(N_{R}\right)}\left(\sum_{d \mid N / N_{R}} \frac{\left|D_{d}\right|}{d}\right)^{\sigma\left(N_{R}\right)} \prod_{d \mid N_{R}}\left|I_{d}\right| \prod_{d \mid N_{R}} \frac{\left|D_{d}\right|}{d}
$$

where $\sigma\left(N_{R}\right)$ denotes the number of divisors of $N_{R}$. This finishes the proof of the theorem.

Proposition 5.3 and Theorem 5.4 imply the following.

Corollary 5.5. Let $D$ be a discriminant form of square-free level $N$, and let $F$ be a modular form for $\rho_{D}$ which is invariant under $O(D)$. Then $F=F_{\Gamma_{0}(N), f, 0}$ for a suitable modular form $f$ on $\Gamma_{0}(N)$ with character $\chi_{D}$.

This result (Corollary 5.5) is also stated as Theorem 4.2.17 in [1]. However, the proof given there is incomplete because the author does not show that the matrix $A$ is invertible.

\section{$\S 6$. Some new generalized Kac-Moody algebras}

In this section we use the above results to construct some modular forms for the Weil representation with nonnegative integral coefficients and reflective poles. The theta lifts of these modular forms are holomorphic automorphic products of singular weight. They give new examples of generalized Kac-Moody algebras with automorphic denominator identity.

The automorphic forms of this section are related to automorphisms of the Leech lattice with cycle shapes $2^{12}, 3^{8}$, and 3.21 . However, we will see that the first two examples can be constructed more naturally from the Niemeier lattices with root systems $D_{12}^{2}$ and $E_{8}^{3}$. 
Table 1: The orbits of $O\left(\sqrt{3} E_{8}\right)$ on the discriminant form of $\sqrt{3} E_{8}$.

\begin{tabular}{|c|c|c|c|}
\hline norm & length & order & name \\
\hline 0 & 1 & 1 & $0_{0}$ \\
\hline 0 & 2240 & 3 & 0 \\
\hline $1 / 3$ & 240 & 3 & $1_{S}$ \\
& 1920 & 3 & $1_{L}$ \\
\hline $2 / 3$ & 2160 & 3 & 2 \\
\hline
\end{tabular}

We have used the computer algebra systems Magma (see [4]) and PARI/ GP (see [11]) in the proof of some statements.

We begin with the case of cycle shape $3^{8}$ because this is the simplest example.

\section{Cycle shape $3^{8}$}

Let $\Lambda$ be the Leech lattice, and let $g$ be an automorphism of $\Lambda$ of cycle shape $3^{8}$. Then the fixed-point sublattice $\Lambda^{g}$ of $g$ is a primitive sublattice of $\Lambda$ isomorphic to $\sqrt{3} E_{8}$.

The lattice $\sqrt{3} E_{8}$ has genus $I I_{8,0}\left(3^{+8}\right)$ and represents the unique class in this genus. The orthogonal group of $\sqrt{3} E_{8}$ acts transitively on the vectors of norm 6,12 , and 18 . The vectors of norm 24 decompose into two orbits of length 240 and 17280 . It follows that $O\left(\sqrt{3} E_{8}\right)$ has five orbits on the discriminant form of $\sqrt{3} E_{8}$, which we describe in Table 1 .

The centralizer $C(g)$ of $g$ in $O(\Lambda)$ does not induce the full orthogonal group of $\Lambda^{g}$. It acts transitively on the vectors of norm 6,12 , and 18 in $\Lambda^{g}$, and the vectors of norm 24 decompose into three orbits of length 240 , 2160, and 15120. The orbit $1_{L}$ in Table 1 splits into two orbits under $C(g)$ which we denote by $1_{L_{S}}$ and $1_{L_{L}}$. They contain 240 and 1680 elements, respectively.

The orthogonal complement $\Lambda^{g \perp}$ of $\Lambda^{g}$ in $\Lambda$ is isomorphic to $A_{2} \otimes E_{8}$.

The lattice $A_{2} \otimes E_{8}$ is 3 -modular and has genus $I I_{16,0}\left(3^{+8}\right)$. The orthogonal group of $A_{2} \otimes E_{8}$ has five orbits on the discriminant form of $A_{2} \otimes E_{8}$ which are described in Table 2. The theta functions of the corresponding cosets are given by

$$
\begin{aligned}
\theta_{0_{0}}(\tau) & =1+720 q^{2}+13440 q^{3}+97200 q^{4}+455040 q^{5}+1714320 q^{6}+\cdots \\
\theta_{0}(\tau) & =6 q+765 q^{2}+12960 q^{3}+97863 q^{4}+463068 q^{5}+1672083 q^{6}+\cdots
\end{aligned}
$$


Table 2: The orbits of $O\left(A_{2} \otimes E_{8}\right)$ on the discriminant form of $A_{2} \otimes E_{8}$.

\begin{tabular}{|c|c|c|c|}
\hline norm & length & order & name \\
\hline 0 & 1 & 1 & $0_{0}$ \\
\hline 0 & 2240 & 3 & 0 \\
\hline $1 / 3$ & 2160 & 3 & 1 \\
\hline $2 / 3$ & 240 & 3 & $2_{S}$ \\
& 1920 & 3 & $2_{L}$ \\
\hline
\end{tabular}

$$
\begin{aligned}
\theta_{1}(\tau) & =45 q^{4 / 3}+2232 q^{7 / 3}+27306 q^{10 / 3}+170064 q^{13 / 3}+\cdots, \\
\theta_{2_{S}}(\tau) & =3 q^{2 / 3}+168 q^{5 / 3}+6009 q^{8 / 3}+51960 q^{11 / 3}+288600 q^{14 / 3}+\cdots, \\
\theta_{2_{L}}(\tau) & =216 q^{5 / 3}+5697 q^{8 / 3}+52920 q^{11 / 3}+287820 q^{14 / 3}+\cdots
\end{aligned}
$$

Under the action of the centralizer $C(g)$, the orbit $2_{L}$ splits into two orbits of length 240 and 1680, which we denote by $2_{L_{S}}$ and $2_{L_{L}}$.

Since $\Lambda^{g}$ is a primitive sublattice of $\Lambda$, we have a natural isomorphism of groups

$$
i: D_{\Lambda^{g}} \rightarrow D_{\Lambda^{g \perp}}
$$

satisfying $i(\gamma)^{2} / 2=-\gamma^{2} / 2$ for all $\gamma \in D_{\Lambda^{g}}$. The map $i$ sends the orbit $1_{S}$ into $2_{L_{S}}$ and $1_{L_{S}}$ into $2_{S}$ because $\Lambda$ has no vectors of norm 2 .

Let $H$ be a lattice with Gram matrix $\left(\begin{array}{rr}-2 & 3 \\ 3 & 0\end{array}\right)$. Then $H$ has genus $I I_{1,1}\left(9^{-1}\right)$ and we can consider $H$ as a sublattice of $I I_{1,1}$ of index 3 . Hence $\theta_{\Lambda^{g \perp}} / 3 \Delta$ induces a modular form $F_{\theta_{\Lambda} \perp / 3 \Delta}$ of weight -4 for the Weil representation of $L=\Lambda^{g} \oplus H$ (see the remarks at the end of Section 2, Theorem 4.1, and the remark at the end of Section 4). We decompose $D_{L}=D_{\Lambda^{g}} \oplus D_{H}$. Then the components of $F_{\theta^{g \perp}} / 3 \Delta$ are given by

$$
F_{\theta_{\Lambda^{g}} / 3 \Delta, \gamma}=\theta_{i\left(\gamma_{\Lambda^{g}}\right)+\Lambda^{g \perp}} / 3 \Delta
$$

if $\gamma=\left(\gamma_{\Lambda^{g}}, \gamma_{H}\right)$ with $\gamma_{H} \in D_{H}^{3}$ and by $F_{\theta_{\Lambda^{g \perp}} / 3 \Delta, \gamma}=0$ otherwise.

The function $\eta_{g}(\tau)=\eta(3 \tau)^{8}$ is a modular form for $\Gamma_{0}(9)$ of weight 4 with trivial character. This function is related to the theta functions by

$$
\left(\theta_{2_{S}}-\theta_{2_{L}}\right) / 3 \Delta(\tau)=1 / \eta_{g}(\tau / 3) .
$$

We decompose

$$
1 / \eta_{g}(\tau / 9)=g_{0}(\tau)+\cdots+g_{8}(\tau)
$$


where $\left.g_{j}\right|_{T}(\tau)=e(j / 9) g_{j}(\tau)$. Note that the only nonvanishing $g_{j}$ are $g_{2}, g_{5}$, and $g_{8}$. Then the lifting of $1 / \eta_{g}$ on the trivial subgroup of $D_{L}$ is given by

$$
F_{\Gamma_{0}(9), 1 / \eta_{g}, 0}=\frac{3}{\eta_{g}} e^{0}-\sum_{\gamma \in D_{L}^{3}} \frac{1}{\eta_{g}} e^{\gamma}+3 \sum_{\gamma \in D_{L}} g_{j_{\gamma}} e^{\gamma}
$$

where $j_{\gamma}$ is defined by $j_{\gamma} / 9=-\gamma^{2} / 2 \bmod 1$ (see Theorem 3.2). Let

$$
F=F_{\theta_{\Lambda} \perp / 3 \Delta}+\frac{1}{3} F_{\Gamma_{0}(9), 1 / \eta_{g}, 0} .
$$

Then we have the following.

THEOREM 6.1. The function $F$ is a modular form for $\rho_{D_{L}}$ of weight -4 . The components of $F$ are given by

$$
\begin{aligned}
F_{0} & =\frac{1}{\eta_{g}}+\frac{1}{3}\left(\frac{\theta_{0_{0}}}{\Delta}-\frac{1}{\eta_{g}}\right) \\
& =q^{-1}+8+108 q+1072 q^{2}+8790 q^{3}+64512 q^{4}+440176 q^{5}+\cdots
\end{aligned}
$$

and

$$
\begin{aligned}
F_{\gamma} & =\frac{1}{3}\left(\frac{\theta_{0_{0}}}{\Delta}-\frac{1}{\eta_{g}}\right) \\
& =8+108 q+1064 q^{2}+8790 q^{3}+64512 q^{4}+440132 q^{5}+\cdots
\end{aligned}
$$

if $\gamma \in D_{L}^{3} \backslash\{0\}$,

$$
F_{\gamma}=\frac{\theta_{0}}{3 \Delta}=2+303 q+11088 q^{2}+225321 q^{3}+3204240 q^{4}+\cdots
$$

if $\gamma^{2} / 2=0 \bmod 1$ and $\gamma \notin D_{L}^{3}$,

$$
F_{\gamma}=\frac{\theta_{2_{S}}}{3 \Delta}=q^{-1 / 3}+80 q^{2 / 3}+3671 q^{5 / 3}+86736 q^{8 / 3}+1365702 q^{11 / 3}+\cdots
$$

if $\gamma^{2} / 2=1 / 3 \bmod 1$ and $\gamma=\left(\gamma_{\Lambda^{g}}, \gamma_{H}\right)$ with $\gamma_{\Lambda^{g}} \in 1_{L_{S}}$,

$$
F_{\gamma}=\frac{\theta_{2_{L}}}{3 \Delta}=72 q^{2 / 3}+3627 q^{5 / 3}+86544 q^{8 / 3}+1364976 q^{11 / 3}+\cdots
$$

if $\gamma^{2} / 2=1 / 3 \bmod 1$ and $\gamma=\left(\gamma_{\Lambda^{g}}, \gamma_{H}\right)$ with $\gamma_{\Lambda^{g}} \in 1_{S} \cup 1_{L_{L}}$,

$$
F_{\gamma}=\frac{\theta_{1}}{3 \Delta}=15 q^{1 / 3}+1104 q^{4 / 3}+31818 q^{7 / 3}+564192 q^{10 / 3}+\cdots
$$


if $\gamma^{2} / 2=2 / 3 \bmod 1$,

$$
F_{\gamma}=g_{8}=q^{-1 / 9}+192 q^{8 / 9}+7704 q^{17 / 9}+164560 q^{26 / 9}+\cdots
$$

if $\gamma^{2} / 2=1 / 9 \bmod 1$,

$$
F_{\gamma}=g_{5}=44 q^{5 / 9}+2464 q^{14 / 9}+62337 q^{23 / 9}+1020416 q^{32 / 9}+\cdots
$$

if $\gamma^{2} / 2=4 / 9 \bmod 1$, and

$$
F_{\gamma}=g_{2}=8 q^{2 / 9}+726 q^{11 / 9}+22528 q^{20 / 9}+417140 q^{29 / 9}+\cdots
$$

if $\gamma^{2} / 2=7 / 9 \bmod 1$.

The Fourier coefficients of the $F_{\gamma}$ are nonnegative rational integers.

The poles of $F$ define a reflection group of $L$ which has a Weyl vector (see [3, Theorems 12.1 and 10.4]). For our purposes, it is more convenient to consider the corresponding dual notions. We define $W$ as the reflection group of the dual lattice $L^{\prime}$ generated by the roots

$$
\begin{aligned}
& \alpha \in L \quad \text { with } \alpha^{2}=2, \\
& \alpha \in L^{\prime} \quad \text { with } \alpha^{2}=2 / 3 \text { and } \alpha_{\Lambda^{g}}+\Lambda^{g} \in 1_{L_{S}}, \\
& \alpha \in L^{\prime} \quad \text { with } \alpha^{2}=2 / 9 .
\end{aligned}
$$

Then $W$ has a Weyl vector $\rho$ in $\mathbb{R} \otimes L$; that is, the simple roots of $W$ are the roots $\alpha$ satisfying $\rho \alpha=-\alpha^{2} / 2$. The vector $3 \rho$ is a primitive norm 0 vector in $L^{\prime}$, and $3 \rho+L$ is in $D_{L}^{3} \backslash\{0\}$. The primitive norm 0 vectors in $H^{\prime}$ are conjugate under $O\left(H^{\prime}\right)$. We can choose any of these vectors for $3 \rho$.

Let

$$
M=L \oplus I I_{1,1} .
$$

Then $F$ defines a modular form for $\rho_{D_{M}}$. The singular theta correspondence (see [3, Theorem 13.3]) maps $F$ to an automorphic form $\Psi$ on the Grassmannian of 2-dimensional negative definite subspaces of $\mathbb{R} \otimes M$. The lattice $M$ has one orbit of primitive norm 0 vectors of level 1 under $O(M)$ and has no primitive norm 0 vectors of level 9 .

THEOREM 6.2. The automorphic form $\Psi$ is holomorphic and has singular weight 4 . The level 1 expansion is given by

$$
e((\rho, Z)) \prod_{\alpha \in L^{\prime+}}(1-e((\alpha, Z)))^{\left[F_{\alpha+L}\right]\left(-\alpha^{2} / 2\right)}=\sum_{w \in W} \operatorname{det}(w) \eta_{g}((w \rho, Z))
$$


Proof. The level 1 product expansion of $\Psi$ is

$$
e((\rho, Z)) \prod_{\alpha \in L^{\prime+}}(1-e((\alpha, Z)))^{\left[F_{\alpha+L}\right]\left(-\alpha^{2} / 2\right)} .
$$

Since $\Psi$ is holomorphic and has singular weight, its Fourier expansion is supported only on norm 0 vectors. Furthermore, $\Psi$ is antisymmetric under the Weyl group $W$ because the roots of $W$ have multiplicity 1 . It follows that $\Psi$ has the sum expansion

$$
\sum_{w \in W} \operatorname{det}(w) e((w \rho, Z)) \prod_{n>0}(1-e((3 n w \rho, Z)))^{8} .
$$

This proves the theorem.

The above identity is also the denominator identity of a generalized KacMoody algebra whose real simple roots are the simple roots of $W$ and whose imaginary simple roots are the positive integral multiples of $3 \rho$ with multiplicity 8 . The root lattice is $L^{\prime}$, and the multiplicity of a root $\alpha$ in $L^{\prime}$ is given by $\left[F_{\alpha+L}\right]\left(-\alpha^{2} / 2\right)$. This Lie algebra can also be constructed by orbifolding the fake monster algebra with a lift of $g$ (see [2], [12]).

We describe a slight variation of the above construction which simplifies some aspects. Let $N$ be the Niemeier lattice with root system $E_{8}^{3}$ (see [6]). Then the direct product $O\left(E_{8}\right)^{3}$ is a normal subgroup of $O(N)$ with quotient $S_{3}$. Let $g$ be a permutation of the three $E_{8}$-components of $N$ of order 3 . Then $g$ has cycle shape $3^{8}$ and fixed-point sublattice $N^{g}$ isomorphic to $\sqrt{3} E_{8}$. The orthogonal complement $N^{g \perp}$ is isomorphic to $A_{2} \otimes E_{8}$. Here the centralizer $C(g)$ of $g$ in $O(N)$ induces the full orthogonal group of $N^{g}$ so that the natural isomorphism $i: D_{N^{g}} \rightarrow D_{N g \perp}$ maps the orbits of the corresponding size into each other. If we construct $F$ as above, we can describe the components entirely in terms of the orbits of $O\left(\sqrt{3} E_{8}\right)$ on $D_{\sqrt{3} E_{8}}$. This also leads to a simpler description of $W$. For example, the roots of norm $2 / 3$ then correspond to the elements in $1_{S}$.

\section{Cycle shape $2^{12}$}

Let $g$ be an automorphism of $\Lambda$ of cycle shape $2^{12}$. Then $\Lambda^{g}$ is a primitive sublattice of $\Lambda$ isomorphic to $\sqrt{2} D_{12}^{+}$.

The lattice $\sqrt{2} D_{12}^{+}$has genus $I I_{12,0}\left(2_{4}^{+12}\right)$, and there are 3 classes in this genus given by $A_{1}^{12}, \sqrt{2} E_{8} \oplus A_{1}^{4}$, and $\sqrt{2} D_{12}^{+}$. The orthogonal group of $\sqrt{2} D_{12}^{+}$ acts transitively on the vectors of norm 4,6 , and 10 . The vectors of length 8 
Table 3: The orbits of $O\left(\sqrt{2} D_{12}^{+}\right)$on the discriminant form of $\sqrt{2} D_{12}^{+}$.

\begin{tabular}{|c|c|c|c|}
\hline norm & length & order & name \\
\hline 0 & 1 & 1 & $0_{0}$ \\
\hline 0 & 1 & 2 & $0_{S}$ \\
& 990 & 2 & $0_{L}$ \\
\hline $1 / 4$ & 1024 & 2 & 1 \\
\hline $1 / 2$ & 132 & 2 & $2_{S}$ \\
& 924 & 2 & $2_{L}$ \\
\hline $3 / 4$ & 1024 & 2 & 3 \\
\hline
\end{tabular}

decompose into two orbits of length 24 and 7920, and the vectors of length 12 decompose into two orbits of length 5280 and 59136. This implies that $O\left(\sqrt{2} D_{12}^{+}\right)$has seven orbits on the discriminant form of $\sqrt{2} D_{12}^{+}$. They are described in Table 3.

The centralizer $C(g)$ of $g$ in $O(\Lambda)$ does not induce the full orthogonal group of $\Lambda^{g}$. It acts transitively on the vectors of norm 4, 6, and 10 in $\Lambda^{g}$ while the vectors of norm 8 decompose into two orbits of length 24 and 7920 , and the vectors of norm 12 decompose into three orbits of length 5280 , 8448, and 50688. Therefore, the orbit $2_{L}$ in Table 3 splits into two orbits under $C(g)$, which we denote by $2_{L_{S}}$ and $2_{L_{L}}$. They contain 132 and 792 elements, respectively.

The orthogonal complement $\Lambda^{g \perp}$ of $\Lambda^{g}$ in $\Lambda$ is $\Lambda^{-g}$ and is also isomorphic to $\sqrt{2} D_{12}^{+}$. The theta functions of the cosets are given by

$$
\begin{aligned}
\theta_{0_{0}}(\tau) & =1+264 q^{2}+2048 q^{3}+7944 q^{4}+24576 q^{5}+64416 q^{6}+\cdots \\
\theta_{0_{S}}(\tau) & =24 q+3808 q^{3}+50448 q^{5}+268224 q^{7}+947896 q^{9}+\cdots \\
\theta_{0_{L}}(\tau) & =8 q+256 q^{2}+1952 q^{3}+8192 q^{4}+25008 q^{5}+62464 q^{6}+\cdots \\
\theta_{1}(\tau) & =24 q^{5 / 4}+464 q^{9 / 4}+2904 q^{13 / 4}+11088 q^{17 / 4}+32032 q^{21 / 4}+\cdots \\
\theta_{2_{S}}(\tau) & =2 q^{2 / 4}+40 q^{6 / 4}+876 q^{10 / 4}+4048 q^{14 / 4}+14650 q^{18 / 4}+\cdots \\
\theta_{2_{L}}(\tau) & =64 q^{6 / 4}+768 q^{10 / 4}+4224 q^{14 / 4}+14848 q^{18 / 4}+40128 q^{22 / 4}+\cdots \\
\theta_{3}(\tau) & =2 q^{3 / 4}+132 q^{7 / 4}+1254 q^{11 / 4}+5964 q^{15 / 4}+19338 q^{19 / 4}+\cdots
\end{aligned}
$$

The lattice $\Lambda^{g}$ is a primitive sublattice of $\Lambda$ so that we have a natural isomorphism of groups

$$
i: D_{\Lambda^{g}} \rightarrow D_{\Lambda^{g \perp}}
$$


Table 4: The orbits of $O\left(\sqrt{2} D_{12}\right)$ on the discriminant form of $\sqrt{2} D_{12}$.

\begin{tabular}{|c|c|c|}
\hline norm & length & order \\
\hline 0 & 1 & 1 \\
\hline 0 & 1 & 2 \\
& 990 & 2 \\
& 990 & 2 \\
& 2 & 2 \\
\hline $1 / 2$ & 132 & 2 \\
& 1848 & 2 \\
& 132 & 2 \\
\hline
\end{tabular}

\begin{tabular}{|c|c|c|}
\hline norm & length & order \\
\hline $1 / 4$ & 24 & 4 \\
& 1584 & 4 \\
& 4096 & 4 \\
& 440 & 4 \\
\hline $3 / 4$ & 440 & 4 \\
& 4096 & 4 \\
& 1548 & 4 \\
& 24 & 4 \\
\hline
\end{tabular}

satisfying $i(\gamma)^{2} / 2=-\gamma^{2} / 2$ for all $\gamma \in D_{\Lambda^{g}}$. Since $\Lambda$ has no roots, $i$ maps the orbit $2_{L_{S}}$ of $D_{\Lambda^{g}}$ into the orbit $2_{S}$ of $D_{\Lambda^{g \perp}}$, and the orbit $2_{S}$ into $2_{L_{S}}$.

Let $K$ be a lattice isomorphic to $\sqrt{2} D_{12}$. Then $K$ has genus $I I_{12,0}\left(2_{I I}^{-10} 4_{I I}^{-2}\right)$. There are 2 classes in this genus and the other class is given by $\sqrt{2}\left(E_{8} \oplus D_{4}\right)$. The group $O(K)$ has 16 orbits on $D_{K}$. We describe them in Table 4.

The lattice $\Lambda^{g}$ contains a unique sublattice isomorphic to $K$. This is the sublattice generated by the 264 vectors of norm 4 in $\Lambda^{g}$.

We consider $K$ as a sublattice of $\Lambda^{g}$. Then $H=\Lambda^{g} / K$ is a subgroup of $D_{K}^{2}$ of order 2 . Note that the elements in $D_{K}$ of norm 0 or $1 / 2$ all have order dividing 2 and therefore are in $H^{\perp}$. The function $\theta_{\Lambda^{g \perp}} / 2 \Delta$ induces a modular form $F_{\theta_{\Lambda} \perp / 2 \Delta}$ of weight -6 for the Weil representation of $K$. The components are given by

$$
F_{\theta_{\Lambda^{g \perp}} / 2 \Delta, \gamma}=\theta_{i(\gamma+H)+\Lambda^{g \perp}} / 2 \Delta
$$

if $\gamma \in H^{\perp}$ and $F_{\theta_{\Lambda} \perp \perp 2 \Delta, \gamma}=0$ otherwise.

The function $\eta_{g}(\tau)=\eta(2 \tau)^{12}$ is a modular form for $\Gamma_{0}(4)$ of weight 6 with trivial character. We have the following relations:

$$
\begin{aligned}
\left(\theta_{0_{0}}-\theta_{0_{S}}\right) / \Delta(\tau) & =1 / \eta_{g}(\tau), \\
\left(\theta_{2_{S}}-\theta_{2_{L}}\right) / 2 \Delta(\tau) & =1 / \eta_{g}(\tau / 2), \\
\left(\theta_{1}+\theta_{3}\right) / 2 \Delta(\tau) & =1 / \eta_{g}(\tau / 4) .
\end{aligned}
$$

We decompose $1 / \eta_{g}(\tau / 4)$ as

$$
1 / \eta_{g}(\tau / 4)=g_{0}(\tau)+g_{1}(\tau)+g_{2}(\tau)+g_{3}(\tau)
$$


with $\left.g_{j}\right|_{T}(\tau)=e(j / 4) g_{j}(\tau)$. Then the lifting of $1 / \eta_{g}$ on the trivial subgroup of $D_{K}$ is given by

$$
F_{\Gamma_{0}(4), 1 / \eta_{g}, 0}=\frac{2}{\eta_{g}} e^{0}-\frac{1}{2} \sum_{\gamma \in D_{K}^{2}} \frac{1}{\eta_{g}} e^{\gamma}+2 \sum_{\gamma \in D_{K}} g_{j_{\gamma}} e^{\gamma}
$$

where $j_{\gamma}$ is defined by $j_{\gamma} / 4=-\gamma^{2} / 2 \bmod 1$. The lift of $1 / \eta_{g}$ on $H$ is

$$
F_{\Gamma_{0}(4), 1 / \eta_{g}, H}=2 \sum_{\gamma \in H} \frac{1}{\eta_{g}} e^{\gamma}-\sum_{\gamma \in D_{K}^{2}} \frac{1}{\eta_{g}} e^{\gamma}+4 \sum_{\gamma \in H^{\perp}} g_{j_{\gamma}} e^{\gamma}
$$

Finally, we define

$$
F=F_{\theta_{\Lambda^{g} \perp} / 2 \Delta}+\frac{1}{2} F_{\Gamma_{0}(4), 1 / \eta_{g}, 0}-\frac{1}{4} F_{\Gamma_{0}(4), 1 / \eta_{g}, H} .
$$

Then the above relations imply the following.

THEOREM 6.3. The function $F$ is a modular form of weight -6 for $\rho_{D_{K}}$. The components of $F$ are given by

$$
\begin{aligned}
F_{0} & =\frac{1}{\eta_{g}}+\frac{1}{2}\left(\frac{\theta_{0_{0}}}{\Delta}-\frac{1}{\eta_{g}}\right) \\
& =q^{-1}+12+300 q+5792 q^{2}+84186 q^{3}+949920 q^{4}+8813768 q^{5}+\cdots
\end{aligned}
$$

and

$$
\begin{aligned}
F_{\gamma} & =\frac{1}{2}\left(\frac{\theta_{0_{0}}}{\Delta}-\frac{1}{\eta_{g}}\right) \\
& =12+288 q+5792 q^{2}+84096 q^{3}+949920 q^{4}+8813248 q^{5}+\cdots
\end{aligned}
$$

if $\gamma \in D_{K}^{2} \backslash\{0\}$

$$
F_{\gamma}=\frac{\theta_{0_{L}}}{2 \Delta}=4+224 q+5344 q^{2}+81792 q^{3}+939232 q^{4}+8769856 q^{5}+\cdots
$$

if $\gamma^{2} / 2=0 \bmod 1$ and $\gamma \notin D_{K}^{2}$,

$$
F_{\gamma}=\frac{\theta_{2_{S}}}{2 \Delta}=q^{-1 / 2}+44 q^{1 / 2}+1242 q^{3 / 2}+22216 q^{5 / 2}+287463 q^{7 / 2}+\cdots
$$

if $\gamma^{2} / 2=1 / 2 \bmod 1$ and $\gamma+H \in 2_{L_{S}}$,

$$
F_{\gamma}=\frac{\theta_{2_{L}}}{2 \Delta}=32 q^{1 / 2}+1152 q^{3 / 2}+21696 q^{5 / 2}+284928 q^{7 / 2}+\cdots
$$


if $\gamma^{2} / 2=1 / 2 \bmod 1$ and $\gamma+H \in 2_{S} \cup 2_{L_{L}}$,

$$
F_{\gamma}=\frac{\theta_{3}}{2 \Delta}=q^{-1 / 4}+90 q^{3 / 4}+2535 q^{7 / 4}+42614 q^{11 / 4}+521235 q^{15 / 4}+\cdots
$$

if $\gamma^{2} / 2=1 / 4 \bmod 1$, and

$F_{\gamma}=\frac{\theta_{1}}{2 \Delta}=12 q^{1 / 4}+520 q^{5 / 4}+10908 q^{9 / 4}+153960 q^{13 / 4}+1669720 q^{17 / 4}+\cdots$

if $\gamma^{2} / 2=3 / 4 \bmod 1$.

The Fourier coefficients of the $F_{\gamma}$ are nonnegative rational integers.

Now let

$$
L=K \oplus I I_{1,1}
$$

We can consider $F$ as a modular form for $\rho_{D_{L}}$. Then the poles of $F$ define a reflection group $W$ of $L^{\prime}$ which has a Weyl vector. The roots of $W$ are the vectors

$$
\begin{aligned}
& \alpha \in L \quad \text { with } \alpha^{2}=2, \\
& \alpha \in L^{\prime} \quad \text { with } \alpha^{2}=1 \text { and }\left(\alpha_{K}+K\right)+H \in 2_{L_{S}}, \\
& \alpha \in L^{\prime} \quad \text { with } \alpha^{2}=1 / 2 .
\end{aligned}
$$

The reflection group $W$ has a Weyl vector $\rho$ in $\mathbb{R} \otimes L$; that is, the simple roots of $W$ are the roots $\alpha$ satisfying $\rho \alpha=-\alpha^{2} / 2$. The vector $2 \rho$ is a primitive norm 0 vector in $L^{\prime}$ and $2 \rho+L$ is in $D_{L}^{2} \backslash\{0\}$.

Let

$$
M=L \oplus I I_{1,1}
$$

Then $F$ defines a modular form for $\rho_{D_{M}}$. Let $\Psi$ be the theta lift of $F$. The lattice $M$ has one orbit of primitive norm 0 vectors of level 1 under $O(M)$ and has no primitive norm 0 vectors of level 4 .

THEOREM 6.4. The automorphic form $\Psi$ is holomorphic and has singular weight 6. The level 1 expansion is given by

$$
e((\rho, Z)) \prod_{\alpha \in L^{\prime+}}(1-e((\alpha, Z)))^{\left[F_{\alpha+L}\right]\left(-\alpha^{2} / 2\right)}=\sum_{w \in W} \operatorname{det}(w) \eta_{g}((w \rho, Z)) .
$$


As in the previous case, this identity is also the denominator identity of a generalized Kac-Moody algebra whose real simple roots are the simple roots of $W$ and whose imaginary simple roots are the positive integral multiples of $2 \rho$ with multiplicity 12 . The root lattice is $L^{\prime}$, and the multiplicity of a root $\alpha$ in $L^{\prime}$ is given by $\left[F_{\alpha+L}\right]\left(-\alpha^{2} / 2\right)$. This Lie algebra can also be constructed by orbifolding the fake monster algebra with a lift of $g$.

We show now that the Niemeier lattice $N$ with root system $D_{12}^{2}$ gives a simpler construction of the above objects. The quotient $D_{12}^{\prime} / D_{12}$ can be represented by the elements $0=\left(0^{12}\right), s=\left((1 / 2)^{12}\right), c=\left((1 / 2)^{11}(-1 / 2)\right)$, and $v=\left(\begin{array}{ll}0^{11} & 1\end{array}\right)$. Adding the glue vectors $(0,0),(s, s),(c, v)$, and $(v, c)$ to the orthogonal sum $D_{12} \oplus D_{12}$, we obtain an even unimodular lattice $N$ with root system $D_{12}^{2}$. The direct product $O\left(D_{12}^{+}\right)^{2}$ is a normal subgroup of $O(N)$ with quotient $S_{2}$. Define $g \in O(N)$ by $g(x, y)=(y, x)$. Then $g$ has order 2 , and $N^{g}$ and $N^{g \perp}$ are both isomorphic to $\sqrt{2} D_{12}^{+}$. The centralizer $C(g)$ of $g$ in $O(N)$ induces the full orthogonal group of $N^{g}$. Hence the orbits of the corresponding size are mapped into each other under the natural map $i: D_{N^{g}} \rightarrow D_{N^{g \perp}}$. We construct a modular form $F$ as above. Then the components of $F$ depend only on the orbits of $O\left(\sqrt{2} D_{12}\right)$ on $D_{\sqrt{2} D_{12}}$. In particular, the generators of the Weyl group of norm 1 correspond to the elements in the two orbits of length 132 in $D_{K}$.

\section{Cycle shape 3.21}

Let $g$ be an automorphism of the Leech lattice of cycle shape 3.21. Then the fixed-point lattice $\Lambda^{g}$ has genus $I I_{2,0}\left(3^{+2} 7^{-1}\right)$, and a Gram matrix is given by $\left(\begin{array}{ll}6 & 3 \\ 3 & 12\end{array}\right)$. The lattice $\Lambda^{g}$ represents the unique class in this genus. The centralizer of $g$ induces the full orthogonal group of $\Lambda^{g}$ (see also [8]).

The lattice $\Lambda^{g, 3}=\Lambda^{g^{3}} \cap \Lambda^{g \perp}$ has some very nice properties. It is isomorphic to $\left(\begin{array}{ll}2 & 1 \\ 1 & 4\end{array}\right) \otimes A_{2}$, and a Gram matrix is given by

$$
\left(\begin{array}{ll}
2 & 1 \\
1 & 4
\end{array}\right) \otimes\left(\begin{array}{ll}
2 & 1 \\
1 & 2
\end{array}\right)=\left(\begin{array}{llll}
4 & 2 & 2 & 1 \\
2 & 4 & 1 & 2 \\
2 & 1 & 8 & 4 \\
1 & 2 & 4 & 8
\end{array}\right)
$$

The lattice $\Lambda^{g, 3}$ has genus $I I_{4,0}\left(3^{+2} 7^{-2}\right)$. It is strongly modular, that is, invariant up to isomorphism under the Atkin-Lehner involutions $W_{d}, d \mid 21$. There are three classes in the genus of $\Lambda^{g, 3}$ with minima 2, 2, and 4; that is, $\Lambda^{g, 3}$ represents the unique class without roots. The orthogonal group of $\Lambda^{g, 3}$ 
Table 5: The orbits of $O\left(\Lambda^{g, 3}\right)$ on $D_{\Lambda^{g, 3}, 3}$

\begin{tabular}{|c|c|c|c|}
\hline norm & length & order & name \\
\hline 0 & 1 & 1 & $0_{0}$ \\
\hline $1 / 3$ & 4 & 3 & 1 \\
\hline $2 / 3$ & 2 & 3 & $2_{S}$ \\
& 2 & 3 & $2_{L}$ \\
\hline
\end{tabular}

has forty orbits on the discriminant form $D_{\Lambda^{g, 3}}$ and four orbits on $D_{\Lambda^{g, 3}, 3}$. They are described in Table 5.

The corresponding theta functions are given by

$$
\begin{aligned}
\theta_{0_{0}}(\tau) & =1+6 q^{2}+12 q^{4}+12 q^{5}+6 q^{6}+24 q^{7}+18 q^{8}+24 q^{10}+\cdots \\
\theta_{1}(\tau) & =3 q^{4 / 3}+6 q^{7 / 3}+6 q^{10 / 3}+6 q^{13 / 3}+12 q^{16 / 3}+6 q^{19 / 3}+\cdots \\
\theta_{2_{S}}(\tau) & =3 q^{2 / 3}+3 q^{8 / 3}+6 q^{11 / 3}+18 q^{14 / 3}+12 q^{17 / 3}+12 q^{20 / 3}+\cdots \\
\theta_{2_{L}}(\tau) & =6 q^{5 / 3}+6 q^{8 / 3}+15 q^{14 / 3}+6 q^{17 / 3}+18 q^{20 / 3}+12 q^{23 / 3}+\cdots
\end{aligned}
$$

We have

$$
\left(\theta_{2_{S}}-\theta_{2_{L}}\right) / 3 \eta_{g^{3}}(\tau)=1 / \eta_{g}(\tau / 3)
$$

Since $\Lambda^{g, 3}$ is a primitive sublattice of the Leech lattice, the theta function of $\Lambda^{g, 3}$ defines a modular form $F$ for the Weil representation of the orthogonal complement $\Lambda^{g, 3 \perp}$ of $\Lambda^{g, 3}$ in $\Lambda$. This lattice has genus $I I_{20,0}\left(3^{+2} 7^{-2}\right)$. In particular, there are no nontrivial isotropic elements in $D_{\Lambda^{g, 3 \perp}, 3}$. This implies that the function $F$ cannot be written as a linear combination of liftings from $\Gamma_{0}(21)$ because such a function would be invariant under the automorphisms of $D_{\Lambda^{g, 3 \perp}, 3}$. But as we have seen above, $F$ does not have this symmetry.

We remark that $\Lambda^{g, 7}=\Lambda^{g^{7}} \cap \Lambda^{g \perp}$ is isomorphic to $\sqrt{3} A_{6}$ and has genus $I I_{6,0}\left(3^{+6} 7^{+1}\right)$. There is exactly one class in this genus.

We write $\theta_{\Lambda^{g, 3}}$ for the scalar-valued theta function of $\Lambda^{g, 3}$. Then $\theta_{\Lambda^{g, 3}}$ is a modular form for $\Gamma_{0}(21)$ of weight 2 with trivial character. We describe the expansions at the cusps. Let $M=\left(\begin{array}{ll}a & b \\ c & d\end{array}\right) \in \Gamma$ such that $c \mid 21, c>0$ and $d=0 \bmod c^{\prime}$, where $c^{\prime}=N / c$. Then

$$
\left.\theta_{\Lambda^{g, 3}}\right|_{M}(\tau)=\epsilon \frac{1}{c^{\prime}} \theta_{\Lambda^{g, 3}}\left(\tau / c^{\prime}\right)
$$

with $\epsilon=\left(\frac{c^{\prime}}{2}\right)$. 
Let $K=\Lambda^{g} \oplus \sqrt{7} I I_{1,1}$. Then $K$ has genus $I I_{3,1}\left(3^{+2} 7^{+3}\right)$. The quotient $\theta_{\Lambda^{g, 3}} / \eta_{g^{3}}$, where $\eta_{g^{3}}(\tau)=\eta(\tau)^{3} \eta(7 \tau)^{3}$, is a modular form for $\Gamma_{0}(21)$ of weight -1 with character $\chi(M)=\left(\frac{d}{7}\right)$ for $M=\left(\begin{array}{ll}a & b \\ c & d\end{array}\right) \in \Gamma_{0}(21)$. We have

$$
\begin{aligned}
\theta_{\Lambda^{g, 3}}(\tau) / \eta_{g^{3}}(\tau) & =q^{-1}+3+15 q+40 q^{2}+117 q^{3}+288 q^{4}+677 q^{5}+\cdots, \\
\theta_{\Lambda^{g, 3}}(\tau / 3) / \eta_{g^{3}}(\tau) & =q^{-1}+6 q^{-1 / 3}+3+12 q^{1 / 3}+30 q^{2 / 3}+15 q+\cdots .
\end{aligned}
$$

The lift of $\theta_{\Lambda^{g, 3}} / \eta_{g^{3}}$ on $K$ with trivial support is given by

$$
\begin{aligned}
& F_{\Gamma_{0}(21), \theta_{\Lambda} g, 3} / \eta_{g^{3}}, 0 \\
& \quad=\frac{\theta_{\Lambda^{g, 3}}}{\eta_{g^{3}}} e^{0}+\frac{1}{3} \sum_{\gamma \in D_{K, 3}} g_{3, j_{\gamma}} e^{\gamma}+\sum_{\gamma \in D_{K, 7}} g_{7, j_{\gamma}} e^{\gamma}+\frac{1}{3} \sum_{\gamma \in D_{K}} g_{21, j_{\gamma}} e^{\gamma},
\end{aligned}
$$

where the $g_{c, j}$ are $T$-eigenfunctions with eigenvalue $e(j / c)$ defined by the following decompositions:

$$
\begin{aligned}
\theta_{\Lambda^{g, 3}}(\tau / 3) / \eta_{g^{3}}(\tau) & =g_{3,0}(\tau)+g_{3,1}(\tau)+g_{3,2}(\tau), \\
\theta_{\Lambda^{g, 3}}(\tau / 7) / \eta_{g^{3}}(\tau / 7) & =g_{7,0}(\tau)+g_{7,1}(\tau)+\cdots+g_{7,6}(\tau), \\
\theta_{\Lambda^{g, 3}}(\tau / 21) / \eta_{g^{3}}(\tau / 7) & =g_{21,0}(\tau)+g_{21,1}(\tau)+\cdots+g_{21,20}(\tau) .
\end{aligned}
$$

Note that

$$
\begin{aligned}
\theta_{\Lambda^{g, 3}} / \eta_{g^{3}} & =g_{3,0}, \\
g_{7, j} & =g_{21,3 j} .
\end{aligned}
$$

The functions with poles are $\theta_{\Lambda^{g, 3}} / \eta_{g^{3}}=g_{3,0}$ and

$$
\begin{aligned}
g_{3,2} & =6 q^{-1 / 3}+30 q^{2 / 3}+108 q^{5 / 3}+306 q^{8 / 3}+834 q^{11 / 3}+\cdots, \\
g_{21,20} & =6 q^{-1 / 21}+9792 q^{20 / 21}+835008 q^{41 / 21}+28697184 q^{62 / 21}+\cdots, \\
g_{7,6} & =g_{21,18}=q^{-1 / 7}+1494 q^{6 / 7}+143829 q^{13 / 7}+5254648 q^{20 / 7}+\cdots .
\end{aligned}
$$

For $c \mid 63$, we define the functions $h_{c}(\tau)=1 / \eta_{g}(\tau / c)$ and their $T$ eigenfunctions $h_{c, j}$ with $\left.h_{c, j}\right|_{T}(\tau)=e(j / c) h_{c, j}(\tau)$.

The group $D_{K, 3}$ is generated by two orthogonal elements $\gamma_{1}, \gamma_{2}$ of norm $\gamma_{1}^{2} / 2=\gamma_{2}^{2} / 2=1 / 3 \bmod 1$. The function $h_{3}$ is a modular form of weight -1 
for $\Gamma_{1}(21)$ with character $\chi(M)=e(-b / 3)$ for $M=\left(\begin{array}{ll}a & b \\ c & d\end{array}\right) \in \Gamma_{1}(21)$. The lift of $h_{3}$ on $\gamma_{1}$ is given by (see Theorem 3.7)

$$
\begin{aligned}
\frac{1}{12} F_{\Gamma_{1}(21), h_{3}, \gamma_{1}}= & h_{3}\left(e^{\gamma_{1}}+e^{-\gamma_{1}}\right)-h_{3}\left(e^{\gamma_{2}}+e^{-\gamma_{2}}\right) \\
& -\sum_{\gamma \in \gamma_{1}+D_{K, 7}} h_{21, j_{\gamma}}\left(e^{\gamma}+e^{-\gamma}\right)+\sum_{\gamma \in \gamma_{2}+D_{K, 7}} h_{21, j_{\gamma}}\left(e^{\gamma}+e^{-\gamma}\right) .
\end{aligned}
$$

We have

$$
\begin{aligned}
& \frac{1}{4} F_{\Gamma_{0}(21), \theta_{\Lambda^{g, 3}} / \eta_{g^{3}}, 0}+\frac{1}{24} F_{\Gamma_{1}(21), h_{3}, \gamma_{1}} \\
& =\frac{1}{4} \frac{\theta_{\Lambda^{g, 3}}}{\eta_{g^{3}}} e^{0}+\frac{1}{12} \sum_{\gamma \in D_{K, 3}{ }_{\gamma^{2} / 2 \neq 1 / 3 \bmod 1} g_{3, j_{\gamma}} e^{\gamma}} \\
& \quad+\frac{1}{12}\left(g_{3,2}+6 h_{3}\right)\left(e^{\gamma_{1}}+e^{-\gamma_{1}}\right) \\
& \quad+\frac{1}{12}\left(g_{3,2}-6 h_{3}\right)\left(e^{\gamma_{2}}+e^{-\gamma_{2}}\right) \\
& \quad+\frac{1}{4} \sum_{\gamma \in D_{K, 7}} g_{7, j_{\gamma}} e^{\gamma} g_{21, j_{\gamma}} e^{\gamma} \\
& \quad+\frac{1}{12} \sum_{\gamma \in D_{K}} \sum_{\gamma \neq 1 / 3+n / 7 \bmod 1}\left(g_{21, j_{\gamma}}-6 h_{21, j_{\gamma}}\right)\left(e^{\gamma}+e^{-\gamma}\right) \\
& \quad+\frac{1}{12} \sum_{\gamma \in \gamma_{1}+D_{K, 7}} \sum_{\gamma \in \gamma_{2}+D_{K, 7}}\left(g_{21, j_{\gamma}}+6 h_{21, j_{\gamma}}\right)\left(e^{\gamma}+e^{-\gamma}\right) .
\end{aligned}
$$

The Fourier expansions of the functions $g_{3,1} / 12,\left(g_{3,2} \pm 6 h_{3}\right) / 12, g_{21,3 j} / 12$, $g_{21,3 j+1} / 12$, and $\left(g_{21,3 j+2} \pm 6 h_{21,3 j+2}\right) / 12$ have nonnegative integral coefficients, for example,

$$
\begin{aligned}
\frac{1}{12} g_{3,1} & =q^{1 / 3}+5 q^{4 / 3}+17 q^{7 / 3}+48 q^{10 / 3}+123 q^{13 / 3}+\cdots \\
& =\frac{\theta_{1}}{3 \eta_{g^{3}}}
\end{aligned}
$$




$$
\begin{aligned}
\frac{1}{12}\left(g_{3,2}+6 h_{3}\right) & =q^{-1 / 3}+3 q^{2 / 3}+10 q^{5 / 3}+27 q^{8 / 3}+72 q^{11 / 3}+\cdots \\
& =\frac{\theta_{2_{S}}}{3 \eta_{g^{3}}}, \\
\frac{1}{12}\left(g_{3,2}-6 h_{3}\right) & =2 q^{2 / 3}+8 q^{5 / 3}+24 q^{8 / 3}+67 q^{11 / 3}+163 q^{14 / 3}+\cdots \\
& =\frac{\theta_{2_{L}}}{3 \eta_{g^{3}}} .
\end{aligned}
$$

Let $M=\Lambda^{g} \oplus \sqrt{7} I I_{1,1} \oplus H$, where $H$ is a lattice with Gram matrix $\left(\begin{array}{cc}-2 & 3 \\ 3 & 0\end{array}\right)$. Then $M$ has genus $I I_{4,2}\left(3^{+2} 9^{-1} 7^{+3}\right)$.

The modular form

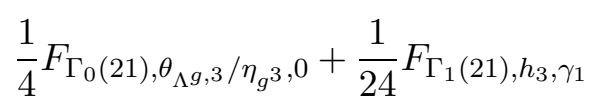

on $K$ induces a modular form $F_{\theta_{\Lambda}, 3} / \eta_{g^{3}}$ on $M$.

We remark that $F_{\theta_{\Lambda}, 3} / \eta_{g^{3}}$ can also be obtained as

$$
F_{\theta_{\Lambda}, 3 / \eta_{g^{3}}}=\frac{1}{12} F_{\Gamma_{0}(63), \theta_{\Lambda} g, 3} / \eta_{g^{3}}, D^{21}+\frac{1}{216} \sum_{\gamma \in \gamma_{1}+D^{21}} F_{\Gamma_{1}(63), h_{3}, \gamma}
$$

The function $\eta_{g}(\tau)=\eta(3 \tau) \eta(21 \tau)$ is a modular form for $\Gamma_{0}(63)$ of weight 1 with character $\chi(M)=\left(\frac{d}{7}\right)$ for $M=\left(\begin{array}{ll}a & b \\ c & d\end{array}\right) \in \Gamma_{0}(63)$. For the lift of $1 / \eta_{g}$ on $M$ with trivial support, we find

$$
\begin{aligned}
& \frac{1}{3} F_{\Gamma_{0}(63), 1 / \eta_{g}, 0} \\
& =\frac{1}{\eta_{g}} e^{0}-\frac{1}{3} \sum_{\gamma \in D^{21}} \frac{1}{\eta_{g}} e^{\gamma}+\sum_{\gamma \in D_{7}} h_{7, j_{\gamma}} e^{\gamma}-\frac{1}{3} \sum_{\gamma \in D^{3}} h_{7, j_{\gamma}} e^{\gamma} \\
& \quad+\sum_{\gamma \in D_{9}} h_{9, j_{\gamma}} e^{\gamma}+\sum_{\gamma \in D} h_{63, j_{\gamma}} e^{\gamma} .
\end{aligned}
$$

Let

$$
F=F_{\theta_{\Lambda}, 3 / \eta_{g^{3}}}+\frac{1}{3} F_{\Gamma_{0}(63), 1 / \eta_{g}, 0} .
$$

Then $F$ is a modular form of weight -1 on $M$. Using $\theta_{\Lambda^{g, 3}} / \eta_{g^{3}}=g_{3,0}$ and $g_{7, j}=g_{21,3 j}$, we obtain the following result. 
THEOREM 6.5. The function $F$ is given by

$$
\begin{aligned}
& F=\frac{1}{\eta_{g}} e^{0}+\frac{1}{3} \sum_{\gamma \in D^{21}}\left(\frac{\theta_{\Lambda^{g, 3}}}{\eta_{g^{3}}}-\frac{1}{\eta_{g}}\right) e^{\gamma} \\
& +\frac{1}{12} \sum_{\gamma \in D_{3}} g_{3,1} e^{\gamma} \\
& \gamma^{2} / 2=2 / 3 \bmod 1 \\
& +\frac{1}{12} \sum_{\gamma \in \gamma_{1}+D^{21}}\left(g_{3,2}+6 h_{3}\right)\left(e^{\gamma}+e^{-\gamma}\right) \\
& +\frac{1}{12} \sum_{\gamma \in \gamma_{2}+D^{21}}\left(g_{3,2}-6 h_{3}\right)\left(e^{\gamma}+e^{-\gamma}\right) \\
& +\sum_{\gamma \in D_{7}} h_{7, j_{\gamma}} e^{\gamma}+\frac{1}{3} \sum_{\gamma \in D^{3}}\left(g_{7, j_{\gamma}}-h_{7, j_{\gamma}}\right) e^{\gamma} \\
& +\frac{1}{12} \sum_{\gamma \in D_{21}} g_{21, j_{\gamma}} e^{\gamma} \\
& \gamma^{2} / 2=2 / 3+n / 7 \bmod 1 \\
& +\frac{1}{12} \sum_{\gamma \in \gamma_{1}+D^{3}}\left(g_{21, j_{\gamma}}-6 h_{21, j_{\gamma}}\right)\left(e^{\gamma}+e^{-\gamma}\right) \\
& +\frac{1}{12} \sum_{\gamma \in \gamma_{2}+D^{3}}\left(g_{21, j_{\gamma}}+6 h_{21, j_{\gamma}}\right)\left(e^{\gamma}+e^{-\gamma}\right) \\
& +\sum_{\gamma \in D_{9}} h_{9, j_{\gamma}} e^{\gamma} \\
& +\sum_{\gamma \in D} h_{63, j_{\gamma}} e^{\gamma}
\end{aligned}
$$

The coefficients of the components $F_{\gamma}$ of $F$ are nonnegative rational integers.

Note that without the contributions of the lifting of $h_{3}$, the Fourier coefficients of the principal part of $F$ would be rational but not integral so that we could not apply the singular theta correspondence to $F$.

We describe some components of $F$. They are important to understand the properties of the Weyl group and the Weyl vector we define below. 
Let $\gamma$ be an isotropic element in $D$. Then $\gamma$ is in $D^{3}=D^{21} \oplus D_{7}$. We have

$$
\begin{aligned}
F_{\gamma}= & \frac{1}{\eta_{g}}+\frac{1}{3}\left(\frac{\theta_{\Lambda^{g, 3}}}{\eta_{g^{3}}}-\frac{1}{\eta_{g}}\right)+h_{7,0}+\frac{1}{3}\left(g_{7,0}-h_{7,0}\right) \\
= & \frac{1}{\eta_{g}}+\frac{1}{3}\left(\frac{\theta_{\Lambda^{g, 3}}}{\eta_{g^{3}}}-\frac{1}{\eta_{g}}\right)+\frac{1}{7}\left(\frac{\theta_{\Lambda^{g, 7}}}{\eta_{g^{7}}}-\frac{1}{\eta_{g}}\right) \\
& +\frac{1}{21}\left(\frac{\theta_{\Lambda^{g \perp}}}{\Delta}-\frac{\theta_{\Lambda^{g, 7}}}{\eta_{g^{7}}}-\frac{\theta_{\Lambda^{g, 3}}}{\eta_{g^{3}}}+\frac{1}{\eta_{g}}\right) \\
= & q^{-1}+2+1060 q+83728 q^{2}+2790636 q^{3}+57148320 q^{4}+\cdots
\end{aligned}
$$

if $\gamma=0$

$$
\begin{aligned}
F_{\gamma} & =\frac{1}{3}\left(\frac{\theta_{\Lambda^{g, 3}}}{\eta_{g^{3}}}-\frac{1}{\eta_{g}}\right)+\frac{1}{3}\left(g_{7,0}-h_{7,0}\right) \\
& =\frac{1}{3}\left(\frac{\theta_{\Lambda^{g, 3}}}{\eta_{g^{3}}}-\frac{1}{\eta_{g}}\right)+\frac{1}{21}\left(\frac{\theta_{\Lambda^{g \perp}}}{\Delta}-\frac{\theta_{\Lambda^{g, 7}}}{\eta_{g^{7}}}-\frac{\theta_{\Lambda^{g, 3}}}{\eta_{g^{3}}}+\frac{1}{\eta_{g}}\right) \\
& =2+1060 q+83720 q^{2}+2790636 q^{3}+57148320 q^{4}+846724518 q^{5}+\cdots
\end{aligned}
$$

if $\gamma \in D^{21} \backslash\{0\}$,

$$
\begin{aligned}
F_{\gamma} & =h_{7,0}+\frac{1}{3}\left(g_{7,0}-h_{7,0}\right) \\
& =\frac{1}{7}\left(\frac{\theta_{\Lambda^{g, 7}}}{\eta_{g^{7}}}-\frac{1}{\eta_{g}}\right)+\frac{1}{21}\left(\frac{\theta_{\Lambda^{g \perp}}}{\Delta}-\frac{\theta_{\Lambda^{g, 7}}}{\eta_{g^{7}}}-\frac{\theta_{\Lambda^{g, 3}}}{\eta_{g^{3}}}+\frac{1}{\eta_{g}}\right) \\
& =1+1055 q+83714 q^{2}+2790597 q^{3}+57148224 q^{4}+846724377 q^{5}+\cdots
\end{aligned}
$$

if $\gamma \in D_{7} \backslash\{0\}$, and

$$
\begin{aligned}
F_{\gamma} & =\frac{1}{3}\left(g_{7,0}-h_{7,0}\right) \\
& =\frac{1}{21}\left(\frac{\theta_{\Lambda^{g \perp}}}{\Delta}-\frac{\theta_{\Lambda^{g, 7}}}{\eta_{g^{7}}}-\frac{\theta_{\Lambda^{g, 3}}}{\eta_{g^{3}}}+\frac{1}{\eta_{g}}\right) \\
& =1+1055 q+83707 q^{2}+2790597 q^{3}+57148224 q^{4}+846724293 q^{5}+\cdots
\end{aligned}
$$

if $\gamma \in D^{3} \backslash\left\{D^{21} \cup D_{7}\right\}$. 
Next we describe the singular components of $F$. They are given by

$$
\begin{aligned}
F_{0} & =\frac{1}{\eta_{g}}+\frac{1}{3}\left(\frac{\theta_{\Lambda^{g, 3}}}{\eta_{g^{3}}}-\frac{1}{\eta_{g}}\right)+h_{7,0}+\frac{1}{3}\left(g_{7,0}-h_{7,0}\right) \\
& =q^{-1}+2+1060 q+83728 q^{2}+2790636 q^{3}+57148320 q^{4}+\cdots
\end{aligned}
$$

and

$$
\begin{aligned}
F_{\gamma} & =\frac{1}{12}\left(g_{3,2}+6 h_{3}\right)+\frac{1}{12}\left(g_{21,14}-6 h_{21,14}\right) \\
& =q^{-1 / 3}+166 q^{2 / 3}+22071 q^{5 / 3}+925654 q^{8 / 3}+21777668 q^{11 / 3}+\cdots
\end{aligned}
$$

if $\gamma^{2} / 2=1 / 3 \bmod 1$ and $\gamma \in \pm \gamma_{1}+D^{21}$,

$$
\begin{aligned}
F_{\gamma} & =h_{7,6}+\frac{1}{3}\left(g_{7,6}-h_{7,6}\right)+h_{63,54} \\
& =h_{7,6}+\frac{1}{3}\left(g_{7,6}-h_{7,6}\right) \\
& =q^{-1 / 7}+498 q^{6 / 7}+47943 q^{13 / 7}+1751560 q^{20 / 7}+37970952 q^{27 / 7}+\cdots
\end{aligned}
$$

if $\gamma^{2} / 2=1 / 7 \bmod 1$ and $\gamma \in D_{7}$,

$$
\begin{aligned}
F_{\gamma} & =h_{9,8}+h_{63,56} \\
& =q^{-1 / 9}+584 q^{8 / 9}+54268 q^{17 / 9}+1943680 q^{26 / 9}+41603422 q^{35 / 9}+\cdots
\end{aligned}
$$

if $\gamma^{2} / 2=1 / 9 \bmod 1$ and $\gamma \in D_{9}$,

$$
\begin{aligned}
F_{\gamma} & =\frac{1}{12}\left(g_{21,20}+6 h_{21,20}\right)+h_{63,60} \\
& =\frac{1}{12}\left(g_{21,20}+6 h_{21,20}\right) \\
& =q^{-1 / 21}+824 q^{20 / 21}+69660 q^{41 / 21}+2391912 q^{62 / 21}+\cdots
\end{aligned}
$$

if $\gamma^{2} / 2=1 / 21 \bmod 1$ and $\gamma \in \pm \gamma_{2}+D^{3}$, and

$$
\begin{aligned}
F_{\gamma} & =h_{63,62} \\
& =q^{-1 / 63}+960 q^{62 / 63}+78660 q^{125 / 63}+2650432 q^{188 / 63}+\cdots
\end{aligned}
$$

if $\gamma^{2} / 2=1 / 63 \bmod 1$. 
The lattices $\sqrt{7} I I_{1,1} \oplus H$ and $I I_{1,1} \oplus \sqrt{7} H$ are in the same genus and therefore isomorphic. This implies that we can consider $F$ as a modular form on

$$
L=\Lambda^{g} \oplus \sqrt{7} H .
$$

The poles of $F$ define a reflection group $W$ of $L^{\prime}$. The roots of $W$ are the vectors

$$
\begin{aligned}
& \alpha \in L \quad \text { with } \alpha^{2}=2, \\
& \alpha \in L^{\prime} \quad \text { with } \alpha^{2}=2 / 3 \text { and } \alpha+L \in \pm \gamma_{1}+D_{L}^{3}, \\
& \alpha \in L^{\prime} \quad \text { with } \alpha^{2}=2 / 7 \text { and } \alpha+L \in D_{L, 7}, \\
& \alpha \in L^{\prime} \quad \text { with } \alpha^{2}=2 / 9 \text { and } \alpha+L \in D_{L, 9}, \\
& \alpha \in L^{\prime} \quad \text { with } \alpha^{2}=2 / 21 \text { and } \alpha+L \in \pm \gamma_{2}+D_{L}^{3}, \\
& \alpha \in L^{\prime} \quad \text { with } \alpha^{2}=2 / 63 .
\end{aligned}
$$

The reflection group $W$ has a Weyl vector $\rho$ in $\mathbb{R} \otimes L$; that is, the simple roots of $W$ are the roots $\alpha$ satisfying $\rho \alpha=-\alpha^{2} / 2$. The vector $3 \rho$ is a primitive norm 0 vector in $L^{\prime}$, and $3 \rho+L$ is in $D_{L}^{3} \backslash\left(D_{L}^{21} \cup D_{L, 7}\right)$. There is one orbit of primitive norm 0 vectors in $(\sqrt{7} H)^{\prime}$ under the orthogonal group of $(\sqrt{7} H)^{\prime}$, and we can take any vector in this orbit for $3 \rho$.

The function $F$ is a modular form for $M=L \oplus I I_{1,1}$. Let $\Psi$ be the theta lift of $F$. The lattice $M$ has one orbit of primitive norm 0 vectors of level 1 under $O(M)$.

THEOREM 6.6. The automorphic form $\Psi$ is holomorphic and has singular weight 1 . The level 1 expansion is given by

$$
e((\rho, Z)) \prod_{\alpha \in L^{\prime+}}(1-e((\alpha, Z)))^{\left[F_{\alpha+L}\right]\left(-\alpha^{2} / 2\right)}=\sum_{w \in W} \operatorname{det}(w) \eta_{g}((w \rho, Z))
$$

As in the previous cases, this identity is also the denominator identity of a generalized Kac-Moody algebra whose real simple roots are the simple roots of $W$ and whose imaginary simple roots are the positive integral multiples $3 n \rho$ of $3 \rho$ with multiplicity 2 if $7 \mid n$ and multiplicity 1 otherwise. The root lattice is $L^{\prime}$, and the multiplicity of a root $\alpha$ in $L^{\prime}$ is given by $\left[F_{\alpha+L}\right]\left(-\alpha^{2} / 2\right)$. Again, this Lie algebra can also be constructed by orbifolding the fake monster algebra with a lift of $g$. 
Acknowledgments. The author thanks R. E. Borcherds, G. Höhn, and F. Werner for stimulating discussions and helpful comments.

\section{REFERENCES}

[1] A. G. Barnard, The singular theta correspondence, Lorentzian lattices and BorcherdsKac-Moody algebras, Ph.D. dissertation, University of California, Berkeley, Berkeley, Calif., 2003. MR 2705173.

[2] R. E. Borcherds, Monstrous moonshine and monstrous Lie superalgebras, Invent. Math. 109 (1992), 405-444. MR 1172696. DOI 10.1007/BF01232032.

[3] - Automorphic forms with singularities on Grassmannians, Invent. Math. 132 (1998), 491-562. MR 1625724. DOI 10.1007/s002220050232.

[4] W. Bosma, J. Cannon, and C. Playoust, "The Magma algebra system, I: The user language" in Computational Algebra and Number Theory (London, 1993), J. Symbolic Comput. 24, 1997, 235-265. MR 1484478. DOI 10.1006/jsco.1996.0125.

[5] J. H. Bruinier, Borcherds Products on $O(2, l)$ and Chern Classes of Heegner Divisors, Lecture Notes in Math. 1780, Springer, Berlin, 2002. MR 1903920. DOI $10.1007 / \mathrm{b} 83278$.

[6] J. H. Conway and N. J. A. Sloane, Sphere Packings, Lattices and Groups, 3rd ed., Grundlehren Math. Wiss. 290, Springer, New York, 1999. MR 1662447.

[7] F. Diamond and J. Shurman, A First Course in Modular Forms, Graduate Texts in Math. 228, Springer, New York, 2005. MR 2112196.

[8] K. Harada and M.-L. Lang, On some sublattices of the Leech lattice, Hokkaido Math. J. 19 (1990), 435-446. MR 1078499. DOI 10.14492/hokmj/1381517491.

[9] V. V. Nikulin, Integer symmetric bilinear forms and some of their geometric applications (in Russian), Izv. Akad. Nauk SSSR Ser. Mat. 43 (1979), 111-177; English translation in Math. USSR Izv. 14 (1979), 103-167. MR 0525944.

[10] O. T. O'Meara, Introduction to Quadratic Forms, reprint of the 1973 edition, Classics Math., Springer, Berlin, 2000. MR 1754311.

[11] The PARI Group, Bordeaux, PARI/GP, version 2.3.4, 2008, http://pari.math.u-bordeaux.fr/ (accessed September 16, 2015).

[12] N. R. Scheithauer, On the classification of automorphic products and generalized Kac-Moody algebras, Invent. Math. 164 (2006), 641-678. MR 2221135. DOI 10.1007/s00222-006-0500-5.

[13] - The Weil representation of $\mathrm{SL}_{2}(\mathbb{Z})$ and some applications, Int. Math. Res. Not. IMRN 2009, no. 8, 1488-1545. MR 2496771.

Fachbereich Mathematik

Technische Universität Darmstadt

64289 Darmstadt

Germany

scheithauer@mathematik.tu-darmstadt.de 